\title{
A maresin 1/RORa/12-lipoxygenase autoregulatory circuit prevents inflammation and progression of nonalcoholic steatohepatitis
}

\author{
Yong-Hyun Han, ${ }^{1}$ Kyong-Oh Shin, ${ }^{2}$ Ju-Yeon Kim, ${ }^{1}$ Daulat B. Khadka, ${ }^{3}$ Hyeon-Ji Kim, ${ }^{1}$ Yong-Moon Lee, ${ }^{2}$ Won-Jea Cho, ${ }^{3}$ \\ Ji-Young Cha, ${ }^{4}$ Bong-Jin Lee, ${ }^{1}$ and Mi-Ock Lee ${ }^{1,5,6}$ \\ 'College of Pharmacy, Seoul National University, Seoul, South Korea. ${ }^{2}$ College of Pharmacy, Chungbuk National University, Cheongju, South Korea. ${ }^{3}$ College of Pharmacy, Chonnam National University, \\ Gwangju, South Korea. ${ }^{4}$ Laboratory of Cell Metabolism and Gene Regulation, Lee Gil Ya Cancer and Diabetes Institute, Gachon University, Incheon, South Korea. ${ }^{5}$ Bio-MAX Institute and ${ }^{6}$ Research Institute of \\ Pharmaceutical Sciences, Seoul National University, Seoul, South Korea.
}

\begin{abstract}
Retinoic acid-related orphan receptor $\alpha(\mathrm{ROR} \alpha)$ is considered a key regulator of polarization in liver macrophages that is closely related to nonalcoholic steatohepatitis (NASH) pathogenesis. However, hepatic microenvironments that support the function of $R O R \alpha$ as a polarity regulator were largely unknown. Here, we identified maresin 1 (MaR1), a docosahexaenoic acid (DHA) metabolite with a function of specialized proresolving mediator, as an endogenous ligand of ROR $\alpha$. MaR1 enhanced the expression and transcriptional activity of ROR $\alpha$ and thereby increased the M2 polarity of liver macrophages. Administration of MaR1 protected mice from high-fat diet-induced NASH in a ROR $\alpha$-dependent manner. Surprisingly, ROR $\alpha$ increased the level of MaR1 through transcriptional induction of 12-lipoxygenase (12-LOX), a key enzyme in MaR1 biosynthesis. Furthermore, we demonstrated that modulation of 12-LOX activity enhanced the protective function of DHA against NASH. Together, these results suggest that the MaR1/RORa/12-LOX autoregulatory circuit could offer potential therapeutic strategies for curing NASH.
\end{abstract}

\section{Introduction}

Nonalcoholic steatohepatitis (NASH) is caused by chronic activation of the hepatic proinflammatory immune responses, which leads to progression from simple steatosis to cirrhosis and even to hepatocellular carcinoma (1). Liver macrophages play a key role in the homeostasis of hepatic immune function by releasing cytokines and modulating immune cell response in NASH (2). Among the heterogeneous liver macrophage populations, Kupffer cells are the most important mediator of the pathologic environments of NASH $(2,3)$. Macrophages have different functional phenotypes with proinflammatory M1 polarity and antiinflammatory M2 polarity (4). M1 liver macrophages exacerbate liver injury through production of proinflammatory cytokines, such as tumor necrosis factor $\alpha(\mathrm{TNF}-\alpha)$ and interleukin $1 \beta$ (IL-1 $\beta$ ), but M2 liver macrophages could suppress activation of M1 macrophages by secreting antiinflammatory cytokines, including IL-10 (2). Targeting a polarity switch from M1 to M2 in hepatic macrophages ameliorated the symptoms of NASH in high fat diet-induced (HFDinduced) mouse models $(3,5)$.

The development of NASH is associated with changes in the hepatic fatty acid (FA) composition. The percentage of $\omega^{-3}$ poly-

Related Commentary: p. 1524

Conflict of interest: The authors have declared that no conflict of interest exists. Copyright: (5) 2019 American Society for Clinical Investigation Submitted: August 14, 2018; Accepted: January 24, 2019.

Reference information: J Clin Invest. 2019;129(4):1684-1698.

https://doi.org/10.1172/JCl124219. unsaturated FAs (PUFAs) in hepatic triglycerides (TG) and plasma decreased significantly, but that of saturated FAs increased in NASH patients $(6,7)$. Several clinical investigations and a meta-analysis on patients with nonalcoholic fatty liver diseases (NAFLDs) demonstrated a beneficial effect of $\omega-3$ PUFA (8-10). In contrast, controlled trials of NASH patients did not show any improvement in histological features after treatment with either $\omega-3$ fish oil or PUFA $(11,12)$. The reason for this discrepancy is unclear.

FAs and their metabolites are important microenvironmental milieu that modulate polarization of M1 and M2 macrophages in the liver. Inhibition of intrinsic lipolysis disturbed the polarity switch of the M2 phenotype, which suggests that the supply of FAs is an essential source of M2 activation (13). Specific types of FAs including $\omega-3$ FAs were synthesized in macrophages during the induction and resolution phases of the inflammatory process, which indicates that the polarity switch of macrophages is distinctly FA-dependent $(14,15)$. In particular, docosahexaenoic acid (DHA) promoted antiinflammatory M2 function. Impairment of the biosynthetic pathway of DHA increased the level of proinflammatory $\mathrm{M} 1$ phenotype $(16,17)$. In addition, metabolites of DHA, i.e., specialized proresolving lipid mediators (SPMs) including resolvins, protectins, and maresins (MaR), are involved in the resolution of acute inflammation, which is an important step for orchestrating the immune system. The SPMs are bioactive lipids that lead to high levels of antiinflammatory action in acute inflammatory diseases (18). They skew macrophages toward the M2 phenotype and play diverse functional roles in the control of metabolic diseases (19). In particular, MaR1 treatment attenuated the symptoms of obesity, insulin resistance, and liver steatosis 
$(20,21)$. Moreover, M2 macrophages increase the biosynthesis of specific SPMs such as MaR1 (22). However, the detailed mechanisms that delineate how MaR1 switches macrophage polarity and affects the pathophysiology of metabolic diseases have not yet been established.

The orphan nuclear receptor, retinoic acid-related orphan receptor $\alpha(\mathrm{ROR} \alpha$; NR1F1) is a ligand-dependent transcription factor that regulates diverse target genes involved in lipid metabolism and inflammation (23). The low expression level of ROR $\alpha$ in the liver of human patients with NAFLDs and in those of experimental NASH animal models suggests the potential involvement of ROR $\alpha$ in the pathogenesis of NASH $(24,25)$. Previously, we reported that ROR $\alpha$ protects against the development of NASH by reducing hepatic lipogenesis and oxidative stress, and by enhancing mitochondrial quality control (25-27). We further demonstrated that ROR $\alpha$ promotes the M2 polarization of liver macrophages under HFD challenge and ameliorates NASH symptoms (3). Here, we hypothesized that the hepatic milieu, consisting of diverse lipids and lipid metabolites, affects the function of ROR $\alpha$. To date, cholesterol derivatives, such as cholesterol sulfate, have been identified as ligands that directly bind to $\operatorname{ROR} \alpha$ (28). Similarly, stearic acid was shown to bind and activate ROR $\beta$, a subfamily of ROR that is exclusively expressed in the central nervous system (29). In the present study, we investigated the potential of PUFAs and their metabolites to act as ligands of ROR $\alpha$ and induce M2 polarization of liver macrophages. We identified MaR1 as a new endogenous ligand of ROR $\alpha$ with potential for the prevention and treatment of NASH.

\section{Results}

DHA enhances transcription of ROR $\alpha$ that confers $M 2$ polarity ofliver macrophages. Recently, we reported that ROR $\alpha$ is a key regulator of M2 polarity of liver macrophages (3). Here, we examined whether the antiinflammatory and protective function of DHA against $\mathrm{NASH}$ involved the regulatory role of $\mathrm{ROR} \alpha$ in the M2 polarity switch of liver macrophages. First, we found that DHA treatment of the isolated liver macrophages increased expression of ROR $\alpha$ at both protein and transcript levels (Figure 1A). Liver macrophages isolated from DHA-administered mice exhibited a higher level of ROR $\alpha$ compared with those from control mice (Figure 1B). The liver macrophages from fat-1 transgenic mice, which have a high hepatic DHA content, showed an increased level of ROR $\alpha$ compared with those from WT mice (Figure 1C) (30). DHA induced ROR $\alpha$-dependent M2 polarity shifts in liver macrophages. For example, the $\mathrm{CD} 2 \mathrm{O6}^{+} / \mathrm{CD} 80^{+}$ratio as well as the mRNA level of M2 marker genes including krüppel-like factor 4 (Klf4) was increased by DHA; however, the induction disappeared upon knockdown of $\mathrm{ROR} \alpha$ (Figure 1D and Supplemental Figure 1A; supplemental material available online with this article; https://doi.org/10.1172/ JCI124219DS1.) Consistently, DHA did not change the CD206 ${ }^{+}$ $\mathrm{CD}^{+} 0^{+}$ratio in the liver macrophages that were obtained from myeloid-specific ROR $\alpha$ knockout mice (LysM ${ }^{\mathrm{Cre}}-\mathrm{ROR} \alpha^{\mathrm{f} / \mathrm{fl}} ; \mathrm{ROR} \alpha-$ $\mathrm{MKO}$ ) (Figure 1E). DHA increased hepatic expression of M2 markers in HFD-induced NASH liver, but did not alter in HFDfed ROR $\alpha$-MKO liver (Supplemental Figure 1, B and C). DHA induced transcriptional activity of the reporter gene encoding the ROR $\alpha$ response element (RORE), probably due to the formation of transcriptionally active chromatin structures near the RORE that locates in the promoter of ROR $\alpha$ target gene (Figure $1 \mathrm{~F}$ and Supplemental Figure 1D) (3). However, DHA did not recruit a coactivator peptide to ROR $\alpha$ protein, whereas SR1078, a synthetic agonist of ROR $\alpha$, did, indicating that DHA is not an agonist ligand of ROR $\alpha$ (Figure 1G) (31).

MaR1 is a novel ligand of ROR $\alpha$ that activates M2 polarity shift in liver macrophages. We hypothesized that metabolites of DHA, such as resolvin D1 (RvD1) and MaR1, might activate ROR $\alpha$ in liver macrophages (Figure 2A). Surprisingly, MaR1 increased expression of ROR $\alpha$ at both protein and mRNA levels, whereas RvD1 did not (Figure 2B). MaR1 induced M2 switch in liver macrophages; i.e., it increased the $\mathrm{CD} 206^{+} / \mathrm{CD} \mathrm{O}^{+}$ratio and enhanced expression of M2 marker genes such as Klf4, Arg1, and Cd163 (Figure 2, $\mathrm{C}$ and D). The effect of MaR1 on the M2 polarity switch showed dose- and time-dependency; it required at least $50 \mathrm{nM}$ and 8 hours in our experimental setting (Figure 2, E and F). However, these changes diminished when either ROR $\alpha$ suffered knockdown by shRNA or it was genetically deleted (Figure 2, C, D, and F). RvD1 also increased the $\mathrm{CD} 206^{+} / \mathrm{CD} 0^{+}$ratio, but the increase was not abolished in the ROR $\alpha$-deleted liver macrophages (Figure 2D). Since proresolving actions of MaR1 also include phagocytosis, the phagocytic function of MaR1 was measured in the liver macrophages (18). MaR1 induced phagocytosis at low doses of MaR1 within 1 hour (Supplemental Figure 2A). However, the MaR1induced phagocytosis was also observed in the ROR $\alpha$-deleted liver macrophages, suggesting that the phagocytosis function of MaR1 may not be ROR $\alpha$-dependent (Supplemental Figure 2B).

The reporter gene assays and chromatin immunoprecipitation (ChIP) analysis demonstrated that MaR1 induced transcriptional function of ROR $\alpha$ probably due to the recruitment of p300 and resulting active histone modifications (Figure 3, A and B). In this case, MaR1 induced binding of ROR $\alpha$ to a coactivator $\mathrm{p} 300$, but decreased that of a corepressor, NCoR1 (Figure 3C). The effect of MaR1 was relatively specific to ROR $\alpha$ in that mRNA level and transcriptional activity of other nuclear receptors such as ROR $\beta$, $\mathrm{ROR} \gamma$, peroxisome proliferator-activated receptor (PPAR), retinoid X receptor $\alpha$, and liver X receptor $\alpha$ were not altered by MaR1 treatment (Figure 3, D and E). Other SPMs such as protectin D1, RvD1, RvD2, and RvE1 with a wide range of concentrations, i.e., 10 to $500 \mathrm{nM}$, did not modulate the mRNA levels of either Rora or M2 marker genes (Figure 3F and Supplemental Figure 3A). Surprisingly, surface plasmon resonance (SPR) analysis and fluorescence resonance energy transfer (FRET) assays demonstrated that MaR1 directly bound to ROR $\alpha$ protein, but other SPMs did not. The binding affinity of MaR1 was higher than that of cholesterol sulfate, which is a known ROR $\alpha$ agonist (Figure 4, A and B and Supplemental Figure 3B) (32). Preincubation with a blocking ROR $\alpha$ antibody disturbed the binding signal of $\operatorname{ROR} \alpha$ and coactivator peptides in the FRET assay, indicating the specificity of MaR1ROR $\alpha$ binding (Supplemental Figure 3C). A model based on the structure of ROR $\alpha$ and MaR1 suggested that MaR1 fit well in the ligand binding pocket of ROR $\alpha$ through interaction with Arg370, Tyr290, and Val364, which was comparable to the binding pattern of cholesterol sulfate to ROR $\alpha$ (Figure 4, C and D) (28). To show the importance of these interactions, 2 ROR $\alpha$ mutants, $\mathrm{C} 288 \mathrm{~L}$ and A330L, were examined with respect to their responsiveness to the 
A

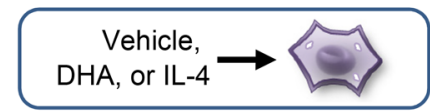

ROR $\alpha$
$F L$ intensity
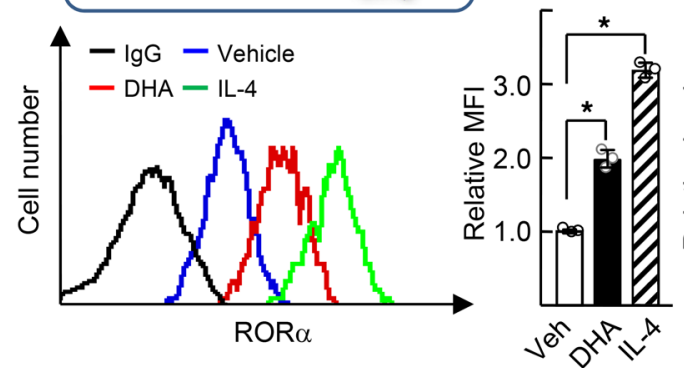

Rora

mRNA

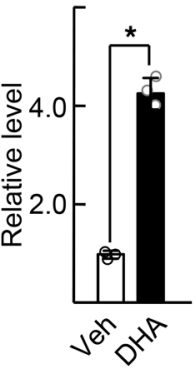

D
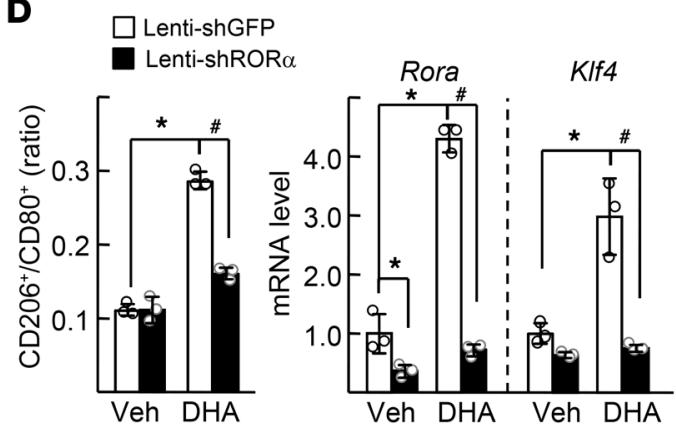

E

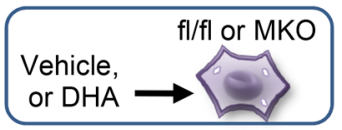

mRNA

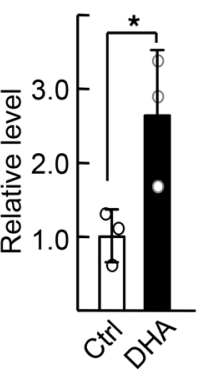

F

RORE-LUC

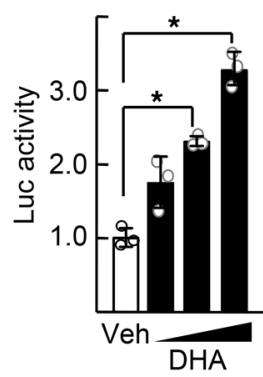

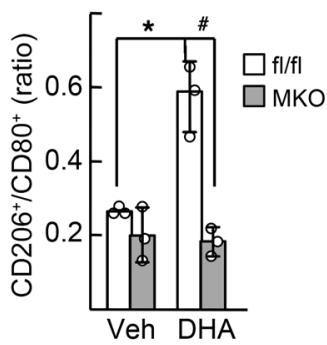

C
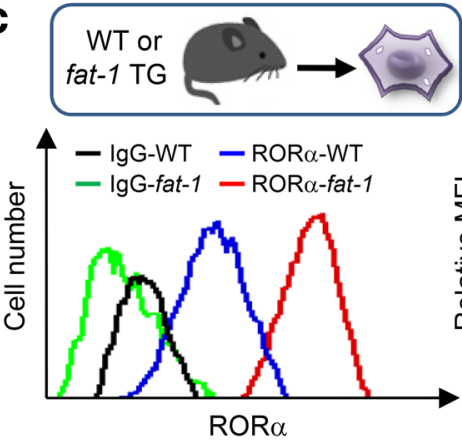

ROR $\alpha$

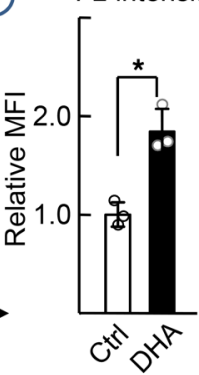

Rora

ROR $\alpha$ $\mathrm{FL}$ intensity

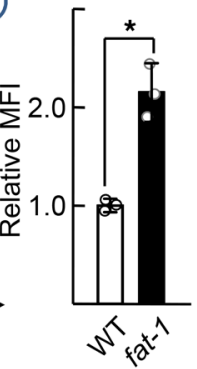
mRNA

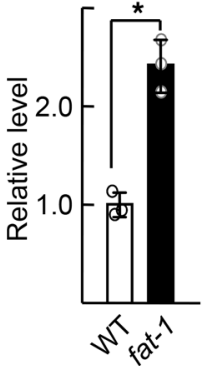

G

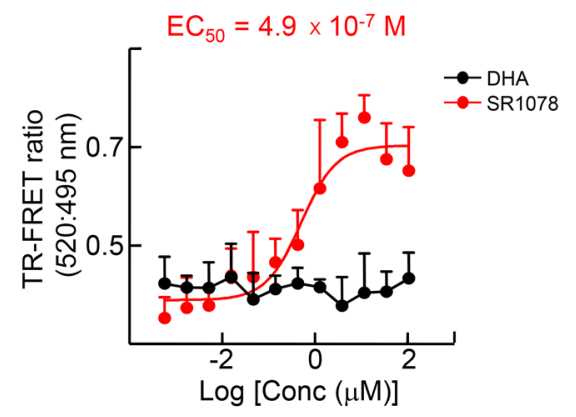

Figure 1. DHA increases expression of ROR $\alpha$ and induces $M 2$ polarity switch in the liver macrophages. (A) The primary culture of liver macrophages was treated with vehicle, $50 \mu \mathrm{M}$ DHA, or $20 \mathrm{ng} / \mathrm{ml} \mathrm{IL-4}$ for 24 hours. (B) The C57BL/6 mice were treated daily with vehicle (Ctrl) or $5 \mathrm{mg} / \mathrm{kg}$ BW DHA by i.p. injection for 5 days, and then the primary liver macrophages were isolated. (C) The primary liver macrophages were isolated from WT or fat-1 transgenic mice. The fluorescence $(\mathrm{FL})$ intensity of stained ROR $\alpha$ protein in the liver macrophages was measured by flow cytometry (left). The numbers represent relative mean fluorescence intensity (MFI) of stained ROR $\alpha$ protein (center). The mRNA level of Rora was measured by qRT-PCR (right). ${ }^{*} P<0.05$ ( $n=3$ ) for A-C. (D) Liver macrophages were infected by lenti-shGFP or lenti-shROR $\alpha$ for 24 hours and then treated with or without $50 \mu M$ DHA for 24 hours. The numbers of $\mathrm{CD} 80^{+} \mathrm{F} 4 / 80^{+}$and the $\mathrm{CD} 206^{+} \mathrm{F} 4 / 80^{+}$macrophages were analyzed by flow cytometry and the CD206 $/ \mathrm{CD} 80^{+}$ratio was determined (left). The mRNA levels of Rora and KIf4 were measured by qRT-PCR (right). (E) The liver macrophages obtained from the livers of floxed and ROR $\alpha$-MKO mice were treated with or without DHA for 24 hours. The $\mathrm{CD} 206^{+} / \mathrm{CD} 80^{+}$ratio of $\mathrm{F} 4 / 80^{+}$cells was determined. ${ }^{*} P<0.05 ;{ }^{\#} P<0.05(n=3)$ for $\mathbf{D}$ and $\mathbf{E}$. (F) Raw 264.7 cells were transfected with the RORE-Luc reporter with Myc-ROR $\alpha$ and then treated with 20, 50, or $100 \mu \mathrm{M}$ DHA for 24 hours. Luciferase activity was measured and normalized by $\beta$-galactosidase activity. ${ }^{*} P<0.05(n=3)$. (G) TR-FRET assay was performed using Lanthascreen ROR $\alpha$ coactivator assay kit. The $y$ axis represents ratio of fluorescence intensity at $520 \mathrm{~nm}$ (signal) and at $495 \mathrm{~nm}$ (background) $(n=4)$. The $x$ axis represents log scale of DHA or SR1078 concentration. The data represent mean \pm SD. Data were analyzed by Mann-Whitney $U$ test for simple comparisons or Kruskal-Wallis test for multiple groups.

MaR1-induced transcriptional activation. As expected, the activities of the ROR $\alpha$ mutants were lower than that of WT, probably due to disruption of the conformation necessary for binding to MaR1 (Figure 4, E and F). Together, these results demonstrate that MaR1 is an agonistic ligand of ROR $\alpha$.

To establish further the involvement of ROR $\alpha$ in the MaR1induced M2 polarity, a global gene expression pattern of the
MaR1-treated liver macrophages was obtained based on microarray analysis. The transcription levels of many genes were altered by MaR1 treatment and the transcriptome profile of MaR1-treated liver macrophages exhibited a strong link to that of IL-4-induced M2 macrophages (Supplemental Figure 4, A and B). Most of the M2 signature genes were upregulated after MaR1 treatment in a similar pattern obtained from the SR1078-treated or the IL-4- 
A

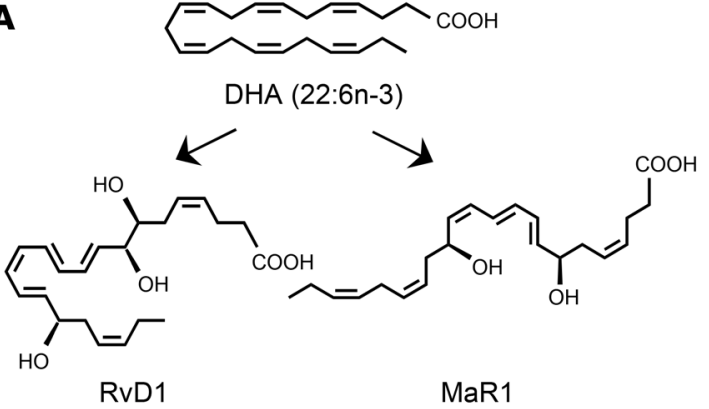

B

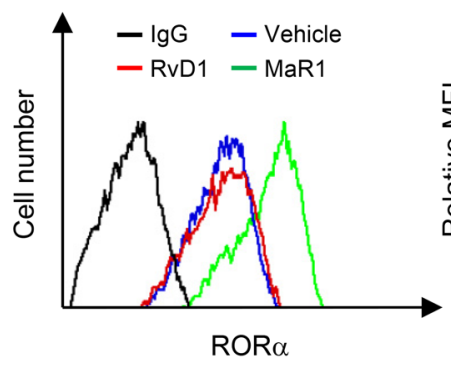

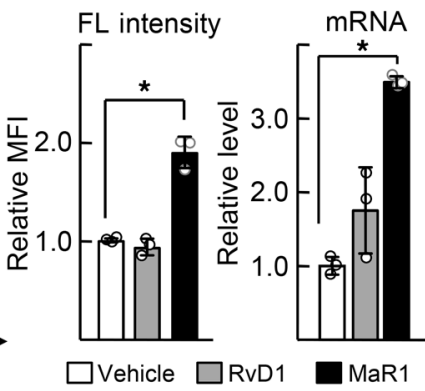
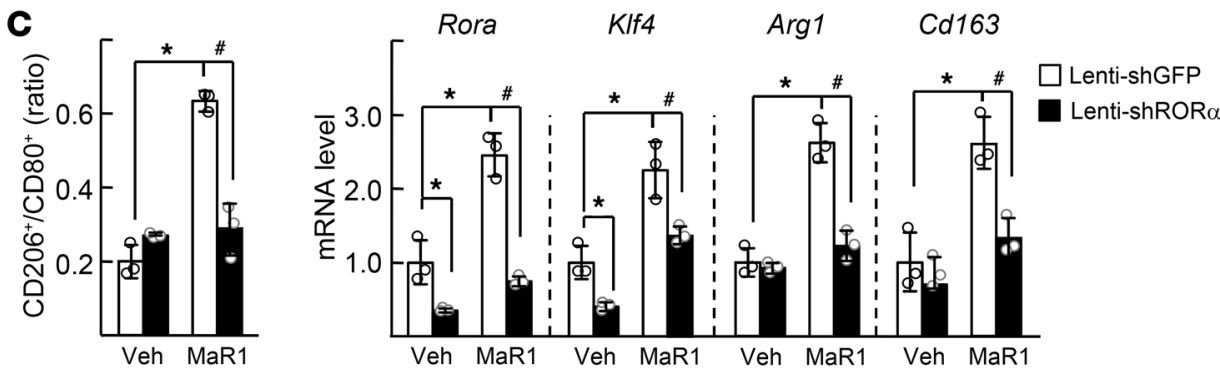

E

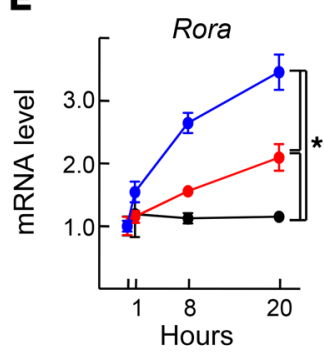

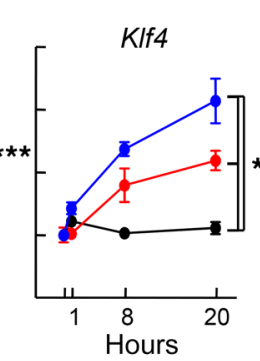

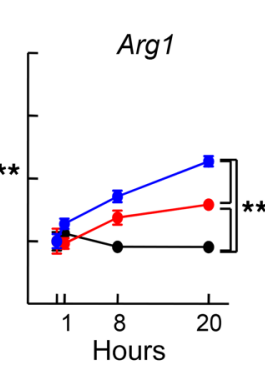

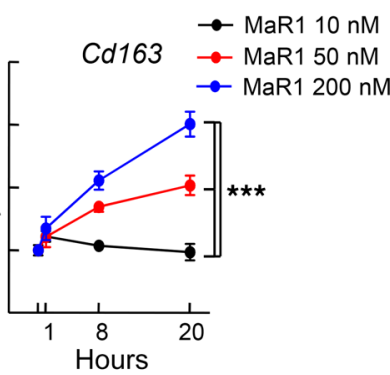

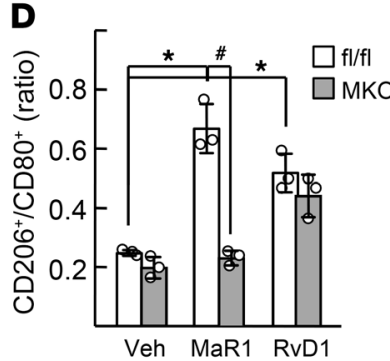

$\mathbf{F}$

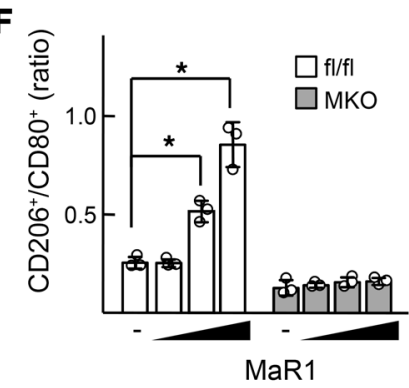

Figure 2. MaR1 enhances expression of ROR $\alpha$ and M2 polarity switch in the liver macrophages. (A) Chemical structures of DHA metabolites RvD1 and MaR1. (B) The primary culture of liver macrophages was treated with 200 nM RvD1 or MaR1 for 24 hours. The FL of RoR $\alpha$ in the liver macrophages was measured by flow cytometry (left). The numbers represent relative MFI of stained ROR $\alpha$ protein (center). The mRNA level of Rora was measured by qRTPCR (right). ${ }^{*} P<0.05(n=3)$. (C) Liver macrophages were infected by lenti-shGFP or lenti-shROR $\alpha$ for 24 hours and then treated with or without 200 nM MaR1 for 24 hours. The CD206 ${ }^{+} / \mathrm{CD} 80^{+}$ratio of F4/80+ cells was determined by flow cytometry (left). The mRNA levels of Rora, KIf4, and M2 marker genes were measured by qRT-PCR (right). (D) The liver macrophages obtained from the livers of floxed and ROR $\alpha$-MKO mice were treated with or without 200 nM MaR1 or RvD1 for 24 hours. The CD206 $/$ CD80+ ratio of F4/80+ cells was determined by flow cytometry. ${ }^{*} P<0.05$; ${ }^{*} P<0.05$ ( $n=3$ ) for $\mathbf{C}$ and $\mathbf{D}$. (E) The liver macrophages were treated with 10, 50, or 200 nM MaR1 for 1, 8, or 20 hours. The mRNA levels of Rora, KIf4, and M2 marker genes were measured by qRTPCR. (F) The liver macrophages obtained from the floxed and the ROR $\alpha$-MKO mice were treated with or without 10,50 , or $200 \mathrm{nM}$ MaR1 for 24 hours. The $\mathrm{CD}^{206^{+}} / \mathrm{CD} \mathrm{C}^{+}$ratio of F4/80+ cells was determined by flow cytometry. ${ }^{*} P<0.05$, and ${ }^{* * *} P<0.001(n=3)$ for $\mathbf{E}$ and $\mathbf{F}$. The data represent mean \pm SD. Data were analyzed by Mann-Whitney $U$ test for simple comparisons or Kruskal-Wallis test for multiple groups.

induced M2 liver macrophages (Supplemental Figure 4, C and D). In silico transcriptional factor analysis showed that the most represented transcription factors in MaR1-treated liver macrophages are $M Z F 1, K l f 4$, and SPI, which are common in both IL-4-treated and SR1078-treated liver macrophages (Supplemental Figure 4E) (3). These data strongly suggest that the MaR1 action requires $\mathrm{ROR} \alpha$ for the M2 polarity shift of the liver macrophages.

MaR1 inhibits progression of HFD-induced NASH in a ROR $\alpha$ dependent manner. We then examined whether MaR1 enhanced the M2 polarity of liver macrophages and thereby improved symptoms of NASH in HFD-fed mice. MaR1 prevented development of NASH in the floxed mice in that it decreased liver weight, hepatic TG level, and liver injury markers, such as serum glutamic pyruvic transaminase (GPT) and glutamic oxaloacetate transaminase (GOT) levels (Figure 5, A and B). As reported previously, the ROR $\alpha-\mathrm{MKO}$ mice showed severe symptoms of NASH. However, these symptoms were not improved by MaR1 administration, indicating that this effect of MaR1 was ROR-dependent (Figure 5, A and B and Supplemental Figure 5). Similarly, fibrotic changes such as collagen deposition, lipid peroxidation, expression of profibrotic proteins, such as $\alpha$-smooth muscle actin ( $\alpha$-SMA), transforming growth factor $\beta$ (TGF- $\beta$ ) and NACHT, LRR, and PYD domain-containing protein 3 (NLRP3), decreased in the MaR1-treated floxed mice, but did not in the MaR1-treated ROR $\alpha$-MKO (Figure 5, C and D and Supplemental Figure 5). MaR1 increased the number of CD206 ${ }^{+} \mathrm{M} 2$ cells in the livers of HFD-fed floxed mice, but did not in the livers of HFD-fed ROR $\alpha$-MKO (Figure 5E). Consistently, the isolated liver macrophages showed the same pattern of M2 polarity changes in flow cytometry analysis (Figure 5F). Expression of the M2 marker genes largely increased by MaR1 administration after HFD feeding 

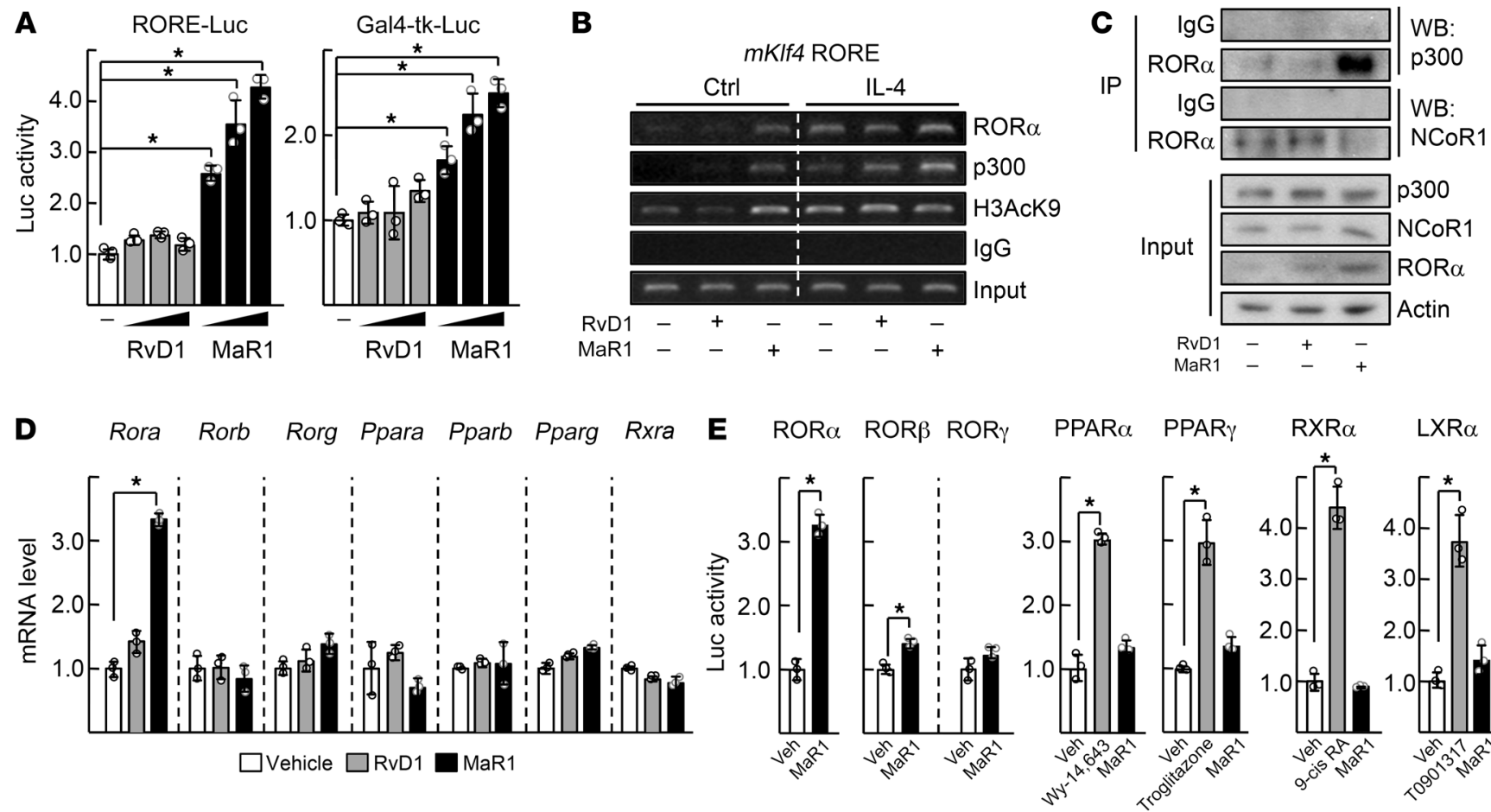

$\operatorname{LXR} \alpha$
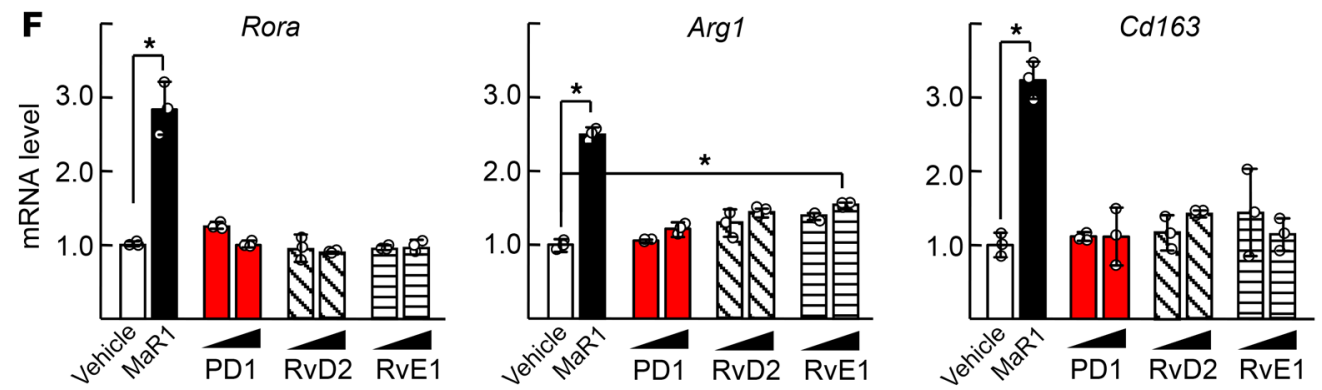

Figure 3. MaR1 is specific for transcriptional activation of RORa. (A) Raw 264.7 cells were transfected with the RORE-Luc reporter with Myc-ROR $\alpha$ (left) or the Gal4-tk-Luc reporter with pM-ROR $\alpha$ (right) and then treated with 100, 200, or $500 \mathrm{nM} \mathrm{RvD1}$ or MaR1 for 24 hours. Luciferase activity was measured and normalized by $\beta$-galactosidase activity. ${ }^{*} P<0.05(n=3)$. (B) Raw 264.7 cells were treated with $200 \mathrm{nM}$ RvD1 or MaR1 in the presence or absence (Ctrl) of $20 \mathrm{ng} / \mathrm{ml} \mathrm{IL-4}$ for 24 hours. DNA fragments that contain flanking region of the ROREs on the Klf4 promoter were immunoprecipitated with indicated antibodies and then amplified by PCR. (C) Whole-cell lysates obtained from the Raw 264.7 cells treated with $200 \mathrm{nM}$ RvD1, or MaR1, were immunoprecipitated (IP) and probed using the indicated antibodies by Western blotting (WB). (D) The liver macrophages were treated with $200 \mathrm{nM}$ RvD1 or MaR1 for 24 hours. The mRNA levels of nuclear receptors were measured by qRT-PCR. (E) Raw 264.7 cells were transfected with overexpression vector of nuclear receptors with corresponding reporters and then treated with $200 \mathrm{nM} \mathrm{MaR} 1$ for 24 hours. Luciferase activity was measured and normalized by $\beta$-galactosidase activity. (F) The liver macrophages were treated with 200 or 500 nM protectin D1 (PD1), RvD2, or RvE1 for 24 hours. For comparison, 200 nM MaR1 was treated. The mRNA levels of Rora, and M2 marker genes were measured by qRT-PCR. ${ }^{*} P<0.05(n=3)$ for $\mathbf{D}-\mathbf{F}$. The data represent mean \pm SD. Data were analyzed by Mann-Whitney $U$ test for simple comparisons.

in control mice, but not in ROR $\alpha$-MKO (Figure 5G). Furthermore, the serum level of TNF- $\alpha$ and IL-10 showed the ROR $\alpha$-dependent M2 polarity switching effect of MaR1 in these mice (Figure $5 \mathrm{H}$ ).

RORa increases biosynthesis of MaR1 by inducing expression of 12-LOX. Interestingly, we found that the hepatic level of MaR1 was lower by $40 \%$ to $60 \%$ in the NASH mice induced by either HFD or methionine choline-deficient (MCD) diet feeding in comparison with the corresponding control mice. The amount of MaR1 was increased by administration of SR1078, but was lowered in the ROR $\alpha$-MKO livers (Figure 6A). The lowered hepatic MaR1 level after HFD feeding in the floxed mice was restored by DHA administration, but not in the ROR $\alpha$-MKO livers (Figure 6B and Supple- mental Figure 1). Surprisingly, the level of MaR1 was increased by SR1078 treatment, but decreased by shROR $\alpha$-induced knockdown in either peritoneal macrophages or Raw 264.7 cells, suggesting that ROR $\alpha$ function may be associated with the level of MaR1 (Figure 6C). It was reported that MaR1 is synthesized by the action of a series of enzymes such as 12-lipoxygenase (12-LOX), 12/15-LOX, and 5-LOX (Figure 6D) (33). We found that expression of 12-LOX encoding platelet type 12-LOX (Alox12) was higher in liver macrophages compared with other types of macrophages or hepatocytes (Figure 6E). The level of Alox12, but not of Alox15 and Alox5, was less in the hepatic macrophages of the ROR $\alpha-\mathrm{MKO}$ compared with that of the floxed mice (Figure 6F). Activation of ROR $\alpha$ by 
A

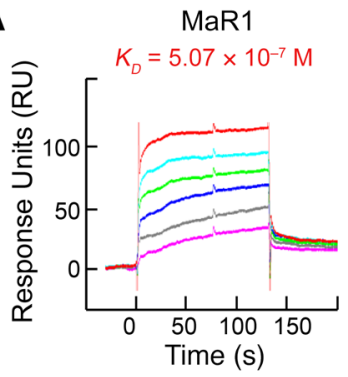

B

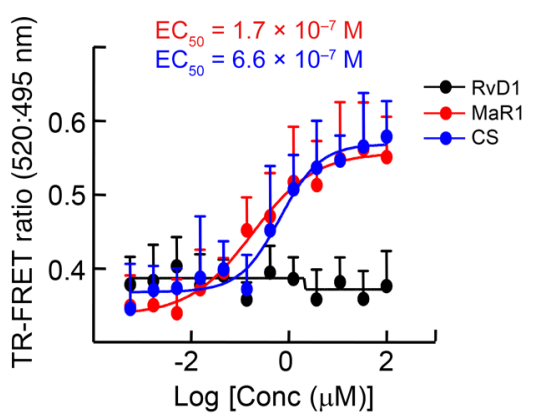

RvD1

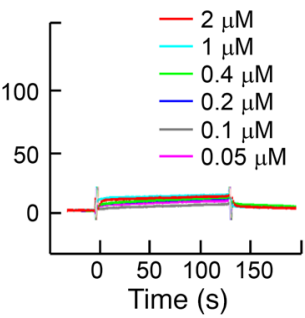

CS

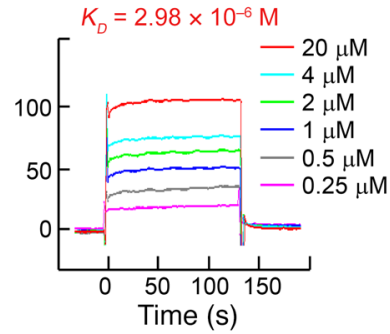

C

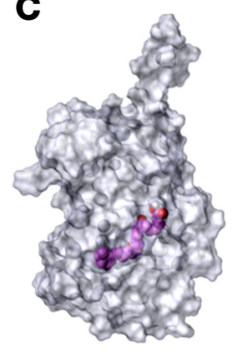

MaR1-bound RORo
D

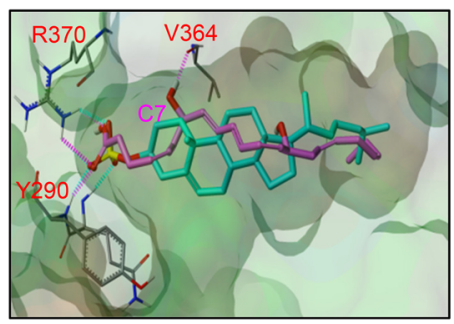

E

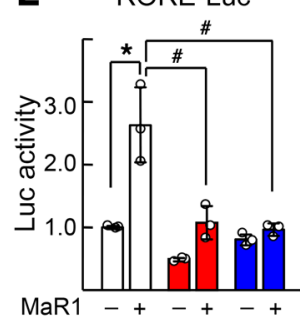

$\square \mathrm{WT}$

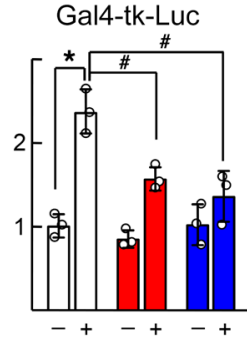

A330L
$\mathbf{F}$

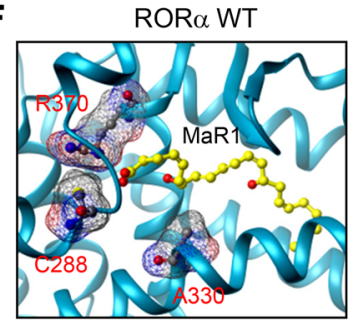

$\mathrm{ROR} \alpha \mathrm{C} 288 \mathrm{~L}$

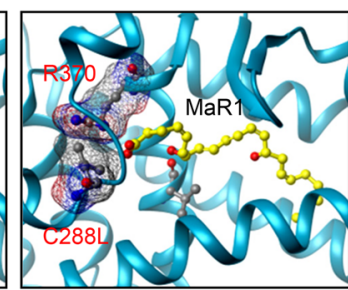

$\mathrm{ROR} \alpha \mathrm{A} 330 \mathrm{~L}$

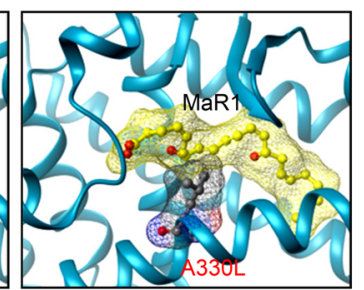

Figure 4. MaR1 is a novel ligand of ROR $\alpha$. (A) BIAcore analysis for binding of MaR1, RvD1, or cholesterol sulfate (CS) to ROR $\alpha$. The increasing concentrations of ligands were injected over immobilized CST-ROR $\alpha$ His proteins on the sensor chip and $K_{D}$ value was calculated by the BIAevaluation 3.1 software. (B) TR-FRET assay was performed using Lanthascreen $\mathrm{ROR} \alpha$ coactivator assay kit. The $y$ axis represents the ratio of fluorescence intensity at $520 \mathrm{~nm}$ (signal) and at $495 \mathrm{~nm}$ (background). The $x$ axis represents log scale of RvD1 (black line), MaR1 (red line), or CS (blue line) concentration. (C) Molecular surface model of ROR $\alpha-M a R 1$ (magenta) complex (transparent view) obtained by docking that allows visualization of ligand bound to the internal ligand binding pocket. The figure was generated using Tripos Benchware 3D Explorer. (D) Docked model showing binding mode of MaR1 (magenta) and cholesterol sulfate (cyan) in the ligand binding pocket of ROR $\alpha$. The $\mathrm{COOH}$ group of MaR1 makes $\mathrm{H}$-bond contacts with $\mathrm{NH} 1-$ Arg370 and $\mathrm{NH}-\mathrm{Ty}$ 290, and $\mathrm{C7}-\mathrm{OH}$ of MaR1 with CO-Val364. (E) Raw 264.7 cells were transfected with the RORE-Luc reporter with the indicated point mutated Myc-ROR $\alpha$ construct (left), or the Gal4-tk-Luc reporter with the indicated point mutated pM-ROR $\alpha$ construct (right). The transfected cells were treated with $200 \mathrm{nM}$ MaR1 for 24 hours and then luciferase activity was measured and normalized by $\beta$-galactosidase activity. ${ }^{*} P<0.05$; $P<0.05(n=3)$. The data represent mean $\pm \mathrm{SD}$. Data were analyzed by Mann-Whitney $U$ test for simple comparisons or Kruskal-Wallis test for multiple groups. (F) A predicted model of interaction between MaR1 (ball \& stick model; carbon atoms in yellow) and native $\mathrm{ROR} \alpha$ (amino acids of interest with meshed molecular surface and ball \& stick model with carbon atoms in gray) (left). Introduction of bulky side chain by the point mutation of C288L would interfere optimal conformation of Arg370 (center). A330L would occupy space necessary for binding MaR1 (carbon atoms in yellow with meshed molecular surface) (right). The figures were generated using Tripos Benchware 3D Explorer. treatment with SR1078, MaR1, or adeno-associated virus (AAV) ROR $\alpha$ increased the level of Alox12 mRNA, whereas knockdown of ROR $\alpha$ decreased, suggesting that Alox 12 may be a ROR $\alpha$ downstream target gene (Figure 6G). We delineated a RORE (-1264 to -1259) in the promoter of mouse Alox12 gene using in silico analysis and subsequent reporter gene and ChIP analysis (Figure 6H). When the activity of 12-LOX was suppressed by known inhibitors such as baicalein and NCTT-956, the SR1078-induced MaR1 level decreased to the basal level (Figure 6I) $(34,35)$. These inhibitors lowered the mRNA level of $\mathrm{ROR} \alpha$ and the $\mathrm{CD} 206^{+} / \mathrm{CD}^{+} 0^{+}$ratio that was induced by DHA, which further suggests the association of ROR $\alpha$ and MaR1 biosynthesis (Figure 6J).

We then examined whether modulation of 12-LOX activity affected the protective effect of DHA on the HFD-induced NASH in mice. First, administration of baicalein abolished the effect of DHA; that is, it worsened symptoms of NASH with increased hepatic TG and serum GPT levels (Figure 7A and Supplemental Figure 6A). Hepatic MaR1 levels that increased after DHA treatment decreased by coadministration with baicalein (Figure 7B). The percentage of M2 polarized liver macrophages also decreased after baicalein treatment (Figure 7C). Second, transduction of AAV-12-LOX together with DHA administration dramatically improved the symptoms of NASH; that is, it decreased the HFD-induced hepatic TG accumulation, serum GPT level, fibrotic lesions, and expression levels of $\alpha$-SMA and TGF- $\beta$ (Figure 7, D-F, and Supplemental Figure 6B). Hepatic MaR1 level and M2 polarity of liver macrophages were consistently higher in the AAV-12LOX-infused group (Figure 7, G and $\mathrm{H}$ ).

Expression of ROR $\alpha$ correlates with the expression of 12-LOX in human patients with NASH. Analysis of the human NCBI Gene Expression Omnibus database (GEO; GSE89632) showed that the hepatic mRNA level of ALOX12 was significantly lower in NASH 
A
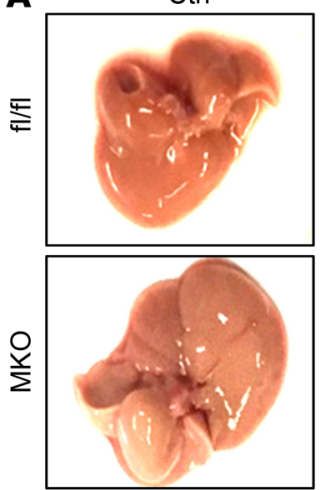

C
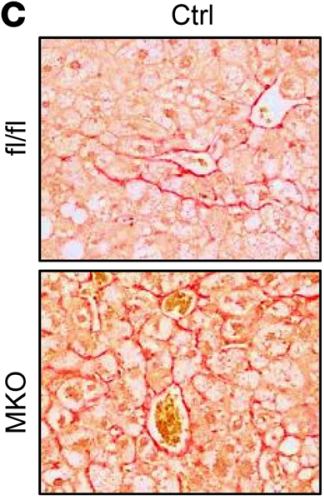

E
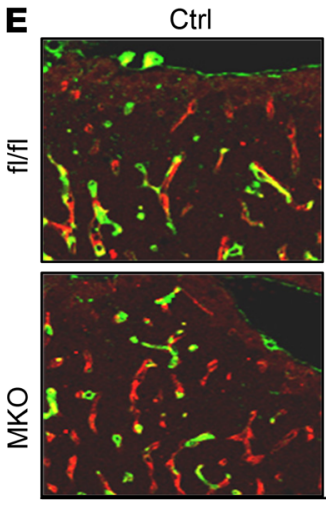

$\mathrm{F} 4 / 80$
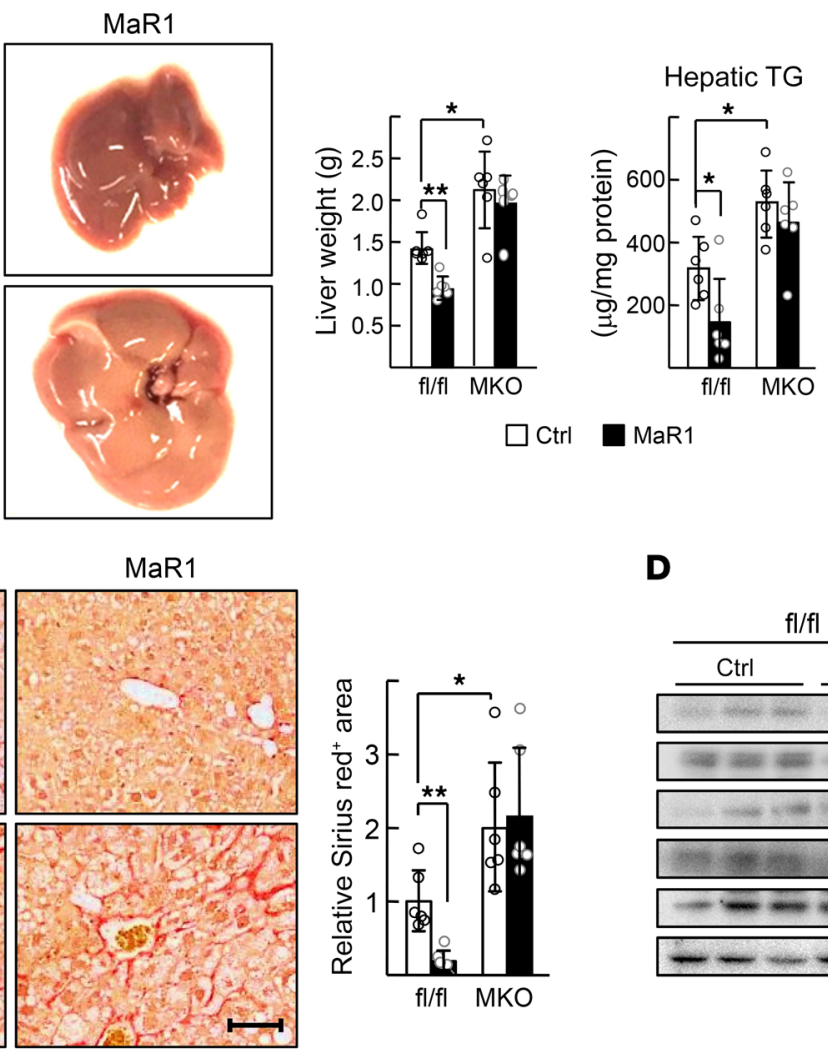

$\square \operatorname{Ctrl} \square$ MaR1
B

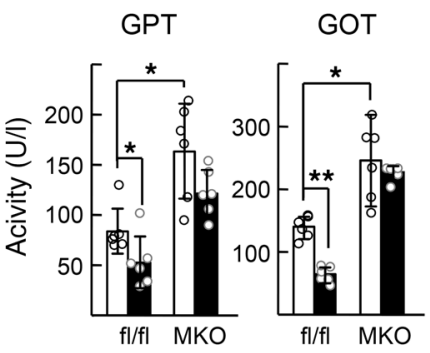

D

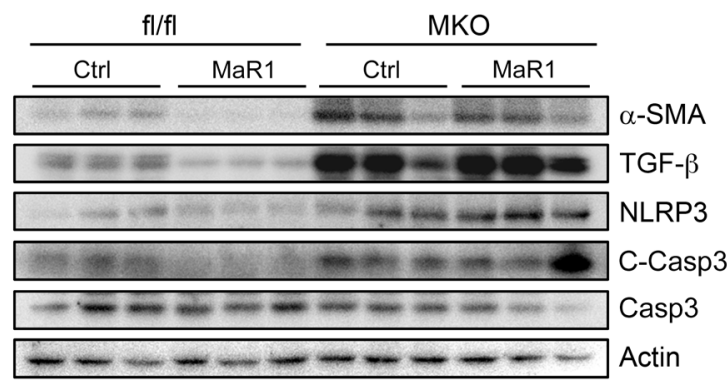

$\mathbf{F}$
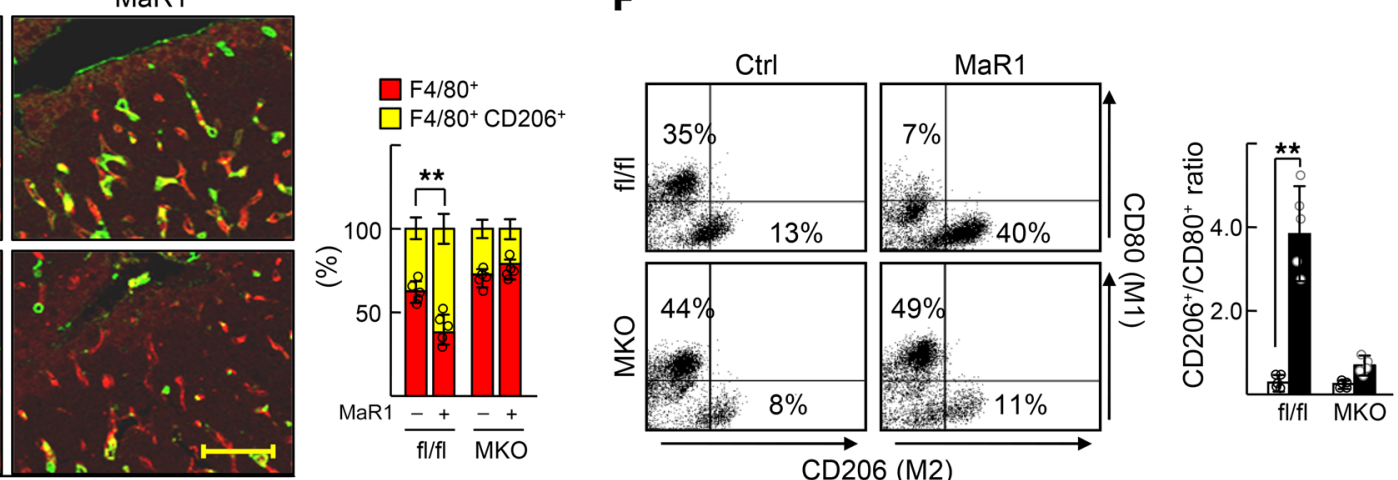

CD206 (M2)

H
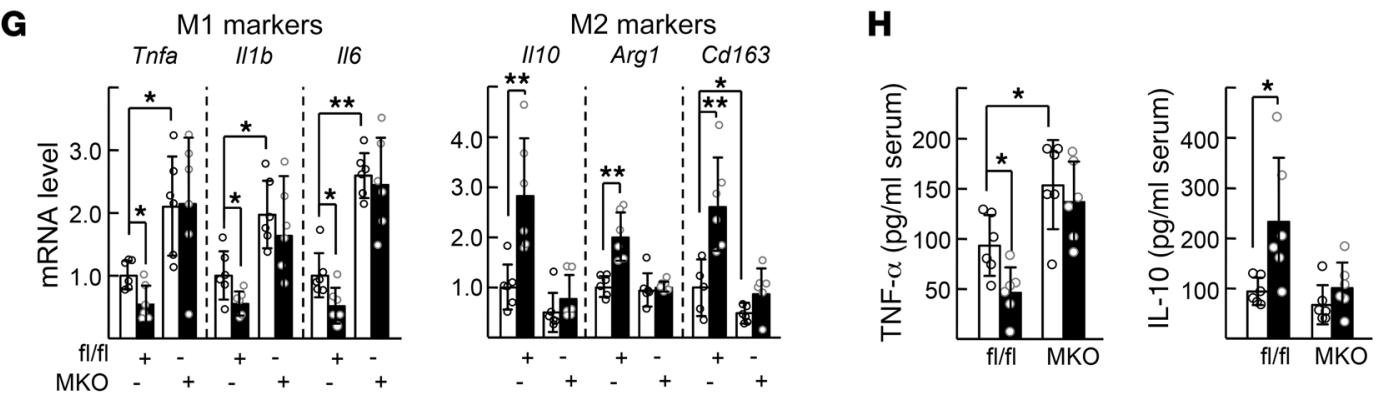

Figure 5. MaR1 improves the HFD-induced NASH in a ROR $\alpha$-dependent manner. Seven-week-old floxed and ROR $\alpha$-MKO mice were fed with HFD for 12 weeks. After 10 weeks of diet feeding, MaR1 was i.p. injected daily at dose of $5 \mu \mathrm{g} / \mathrm{kg}$ BW for 2 weeks. (A) Representative captured livers (left), the liver weights (center), and hepatic TC levels of mice at the end of experiments (right). (B) Serum GPT and GOT activities were analyzed at the end of experiments. (C) Sirius red staining of liver sections (left). Fibrotic area in the liver sections was analyzed by Image J (right). Scale bar: $50 \mu \mathrm{m}$. (D) Levels of $\alpha$-SMA, TCF- $\beta$, NLRP3, and cleaved caspase-3 (C-Casp3) proteins in the liver were analyzed by Western blotting. (E) The expression of F4/80 and CD206 was visualized by red and green immunofluorescence in liver sections (left). The percentage of CD206+ F4/80+ cells was determined by Image J (right). Scale bar: $50 \mu \mathrm{m}$. (F) The $\mathrm{CD}^{2} \mathrm{G}^{+} / \mathrm{CD} 80^{+}$ratio of $\mathrm{F} 4 / 80^{+}$cells was determined by flow cytometry (right). Representative dot plots were shown (left). (C) Hepatic mRNA levels of the M1 and M2 marker genes were measured by GRT-PCR. (H) The concentrations of TNF- $\alpha$ and IL-10 in serum were measured by ELISA. ${ }^{*} P<0.05$ and ${ }^{* *} P<0.01$ ( $n=$ 5-6) for A-H. The data represent mean \pm SD. Data were analyzed by Mann-Whitney $U$ test for simple comparisons or Kruskal-Wallis test for multiple groups. 
patients compared with that in healthy controls. In addition, the transcript levels of RORA and ALOX12 exhibited a positive correlation (Figure 8A). We previously reported that the level of expression of ROR $\alpha$ in liver macrophages was lower in patients with hepatitis compared with that in the controls (3). Similarly, the number of 12-LOX-positive liver macrophages decreased significantly in the liver of chronic hepatitis patients (Figure 8, B and C). Finally, the level of expression of RORA and human ALOX12 in the THP-1 human monocyte/macrophage cell line was induced with both MaR1 and SR1078 treatment (Figure 8D). Together, these results support the relevance of our findings to the clinical pathobiology of NASH.

\section{Discussion}

NASH is a metabolic disease that fails to resolve chronic inflammation induced by lipid-oversupplied steatosis. Despite recent advances in understanding the pathogenesis of NASH, there are no Food and Drug Administration (FDA)-approved pharmacotherapeutics that specifically target NASH. Here, we report that MaR1 acts as an agonistic ligand of ROR $\alpha$ that enhances M2 polarity in liver macrophages and ultimately leads to improvement of the symptoms of NASH (Figure 8E). We also found that RORa increases the biosynthesis of MaR1 from DHA by induction of 12-LOX through an autoregulatory loop of $\mathrm{ROR} \alpha$ activation (Figure 6). The existence of the MaR1/ROR $\alpha / 12-\mathrm{LOX}$ activation circuit could provide a deeper understanding of the mechanism of action of DHA and enable the development of a new strategy that prevents the progression of NASH.

It has been widely accepted that the antiinflammatory and insulin-sensitizing actions of $\omega-3$ PUFAs such as DHA are mediated through their direct binding to the $G$ protein-coupled receptor 120 (GPR120) of adipose tissue macrophages and adipocytes (16). The level of expression of Gpr120 mRNA was lower in visceral adipose tissue of morbidly obese subjects, and it increased by DHA treatment (36). Knock out of GPR120 in mice led to enhanced liver steatosis and insulin resistance after HFD feeding $(16,37)$. $\omega-3$ PUFAs including DHA did not induce M2 marker genes, including Arg1 and Il1O in adipose tissues of these GPR120 knockout mice, indicating the important role of this receptor in the DHA function (16). However, we and others found that expression of GPR120 was extremely low in the liver compared with that in adipose tissue (Supplemental Figure 7, A and B) (38). Neither DHA-induced expression of GPR120 nor knockdown of GPR120 altered the DHA-mediated M2 polarity switch in the liver macrophages (Supplemental Figure 7, C and D). In contrast, the level of expression of the nuclear receptor ROR $\alpha$ was higher in liver macrophages compared with stromal vascular fractions and increased after DHA treatment (Supplemental Figure 7C). Myeloid-specific knockout of ROR $\alpha$ did not affect the production of proinflammatory cytokines, such as TNF- $\alpha$ and IL- $1 \beta$ in adipose tissues, but it increased the level of these cytokines in the liver (3). Together, these results indicate a dual mode of DHA action that is mediated by 2 key receptors in macrophages: GPR120 of adipose tissue macrophages and ROR $\alpha$ of liver macrophages in NAFLD patients. First, the nuclear effect of ROR $\alpha$ in the enhancement of M2 polarity in liver macrophages affects hepatic inflammation and lipid metabolism and second, GPR120-mediated improvement of adipose tissue inflammation results in systemic insulin sensitization.
We demonstrated that 12-LOX, expressed mainly in the liver macrophages, is a key enzyme in the biosynthesis of MaR1 in mouse liver (Figure 6 and Figure 7). Previously, 12/15-LOX was implicated in the catalysis of this reaction in peritoneal exudates of mice $(33,39)$. However, Martínez-Clemente et al. presented unexpected data that showed that dual knockout of APOE and 12/15-LOX improved symptoms of NAFLDs (40). The authors interpreted that a decrease in the level of proinflammatory mediator 12-HETE was involved in the resolution of NAFLD in this case, given that $12 / 15-\mathrm{LOX}$ is also known to convert arachidonic acid into 12-HETE (40, 41). However, lipoxygenases including 12-LOX and 12/15-LOX prefer DHA as a substrate among PUFAs; thus, these enzymes could produce more MaR1 than 12-HETEs if DHA is supplied at sufficient levels (42). Therefore, we speculate that MaR1 was synthesized preferentially by 12 -LOX in the liver macrophages with sufficient supply of DHA in our experimental setting and resulted in improvement of NASH. In addition, we found that the level of expression of $12 / 15$-LOX was much lower than that of 12-LOX in the liver macrophages, further supporting the role of 12-LOX in the liver (Figure 6E). Importantly, transduction of AAV-12-LOX with DHA treatment improved the symptoms of fatty liver injuries by increasing hepatic MaR1 production (Figure 7, D-H). Together, our results suggest that 12-LOX is a key enzyme that improves NAFLD and provides hints for the development of a potential strategy of activating 12-LOX by either small molecules or gene transfer for curing NASH.

MaR1 has previously been shown to regulate macrophage function at much lower concentrations to compare with the present study. For example, $0.1 \mathrm{nM}$ MaR1 enhanced phagocytosis of mouse peritoneal and human peripheral blood mononuclear cellderived macrophages $(39,43)$. In our study, the MaR1-induced M2 polarity switch of the liver macrophages required concentrations higher than $50 \mathrm{nM}$ (Figure 2, E and F). This discrepancy may indicate differential levels of MaR1 in different body fluids and/ or different signaling pathways involved in the MaR1 action. It was reported that concentration of MaR1 ranges from 30-200 pM in mouse peritoneum and is approximately $40-80 \mathrm{pM}$ in human serum $(44,45)$. In contrast, the level of MaR1 in mouse liver seems much higher than that in the peritoneum or blood; we found that the amount of MaR1 in mouse liver ranged from 100-400 pg/mg tissue, which corresponds to $250-1000 \mathrm{nM}$ (Figure 6A). Thus, the hepatic concentrations of MaR1 could be sufficient for activation of ROR $\alpha$ and M2 polarity switch in the liver macrophages. Also, a conflict was found for the action of RvD1 and RvD2 on the M2 polarity switch of macrophages; Titos et al. and Chiang et al. reported that RvD1 and RvD2 increased M2 polarization of peritoneal and peripheral blood mononuclear cell-derived macrophages at nanomolar concentrations, however, we did not observe the increase at wide range of concentrations in the liver macrophages (Figure 3F and Supplemental Figure 3A) $(46,47)$. This discrepancy might be due to the different types of macrophages examined, although further evidence is required to understand.

Proresolving actions of MaR1 consist of a series of reactions including phagocytosis, inhibition of proinflammatory cytokines, and augmentation of M2 polarization, which may require a different duration of exposure (18). MaR1 induced phagocytosis within 1 hour, whereas M2 activation required at least 6 hours longer 

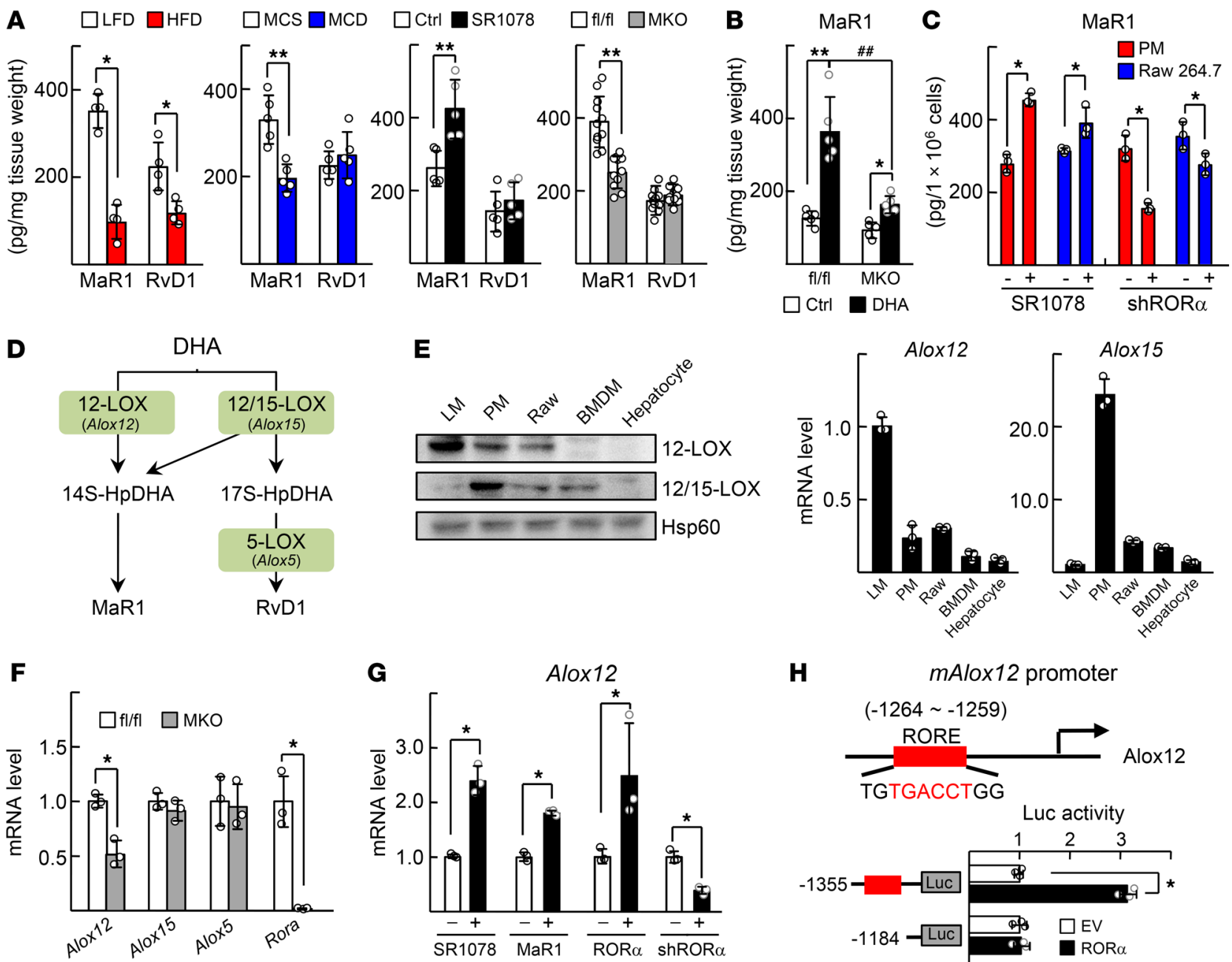

H

mAlox12 promoter
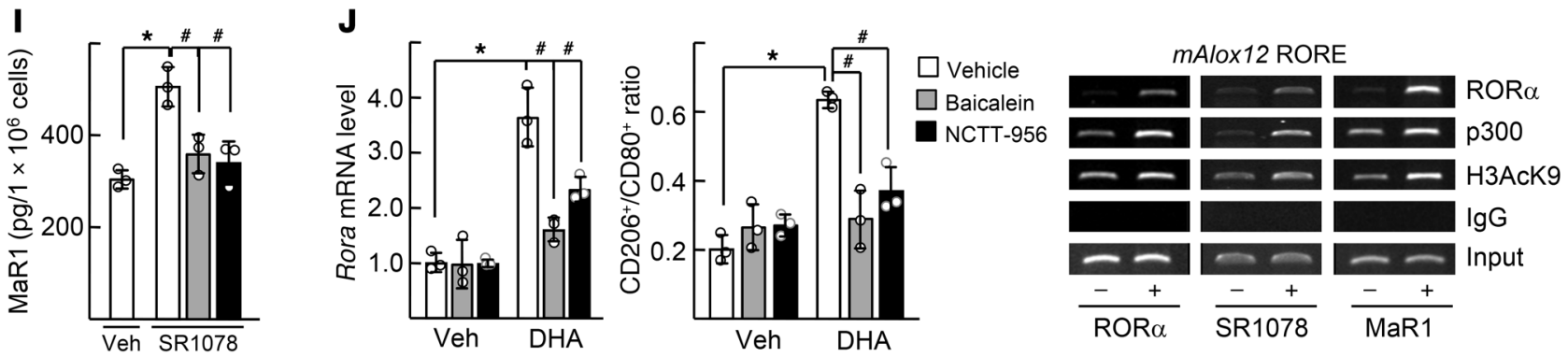

Figure 6. ROR $\alpha$ activates Alox12-dependent MaR1 synthesis. (A) Seven-week-old C57BL/ 6 mice were fed with either LFD or HFD for 12 weeks ( $n=4$ ) or fed with MCS or MCD for 4 weeks ( $n=5$ ) (first and second panels). The LFD-fed C57BL/6 mice were treated with $5 \mathrm{mg} / \mathrm{kg}$ BW SR1078 for 5 days ( $n=5$ ) (third panel). Seven-week-old LFD-fed floxed and ROR $\alpha$-MKO mice were sacrificed $(n=11)$ (fourth panel). (B) Liver samples were obtained from the floxed and ROR $\alpha$-MKO mice those described in Supplemental Figure $1(n=5)$. Levels of MaR1 and RvD1 in liver tissues were measured. ${ }^{*} P<0.05$ and ${ }^{* *} P<0.01 ; \# \# P<$ 0.01 for $\mathbf{A}$ and B. (C) DHA-treated peritoneal macrophages (PM) and Raw 264.7 cells were treated with $5 \mu$ M SR1078 for 24 hours, or the cells were infected by lenti-shGFP or lenti-shROR $\alpha$ for 48 hours. Intracellular amount of MaR1 were measured. ${ }^{*} P<0.05(n=3)$. (D) A scheme for biosynthesis of MaR1 by LOX family. (E) Expression levels of 12-LOX protein (Alox12 mRNA) and 12/15-LOX protein (Alox15 mRNA) in liver macrophages (LM), PM, Raw 264.7, bone marrow-derived macrophages (BMDM), and hepatocytes were measured by Western blotting and qRT-PCR. (F) mRNA levels of Alox genes in the isolated LMs from floxed and ROR $\alpha$-MKO mice as shown in A were measured by qRT-PCR. (C) LMs were treated with SR1078 or MaR1 (left). LMs were infected by AAV-GFP/AAV-ROR $\alpha$ or lenti-shGFP/lenti-shROR $\alpha$ as indicated (right). The mRNA levels of Alox12 were measured by qRT-PCR. ${ }^{*} P<0.05$ ( $n=3$ ) for $\mathbf{F}$ and G. (H) Schematic representation of the mouse Alox12 promoter with the putative ROREs shown as red boxes (top). Raw 264.7 cells were transfected with the deleted Alox12 promoter-Luc reporter with empty vector (EV) or Myc-ROR $\alpha$. Luciferase activity was measured and normalized by $\beta$-galactosidase activity. ${ }^{*} P<0.05(n=3)$ (middle). Raw 264.7 cells were transfected with Myc-ROR $\alpha$, or cells were treated with SR1078 or MaR1. DNA fragments that contain flanking region of the ROREs on the Alox12 promoter were immunoprecipitated with indicated antibodies and then amplified by PCR (bottom). (I) DHA-treated PMs were treated with $5 \mu$ M SR1078, $5 \mu$ M baicalein, or $10 \mu \mathrm{M}$ NCTT-956. Intracellular MaR1 content was measured. (J) LMs were treated with baicalein, or NCTT-956 in the presence or absence of DHA. The mRNA levels of Rora were measured by qRT-PCR (left). The CD206 $/$ CD80+ ratio of F4/80+ cells was determined by flow cytometry (right). ${ }^{*} P<0.05$ and ${ }^{\#} P<0.05(n=3)$ for I and $\mathbf{J}$. The data represent mean \pm SD. Data were analyzed by MannWhitney $U$ test for simple comparisons or Kruskal-Wallis test for multiple groups. 
A

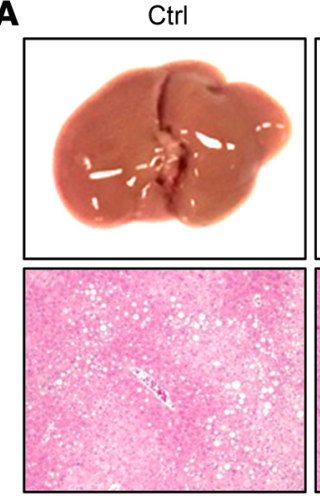

DHA

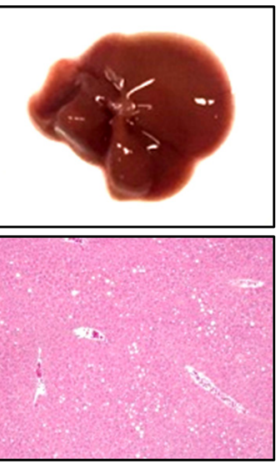

DHA / Baicalein

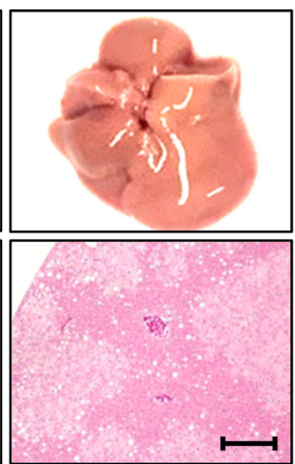

B

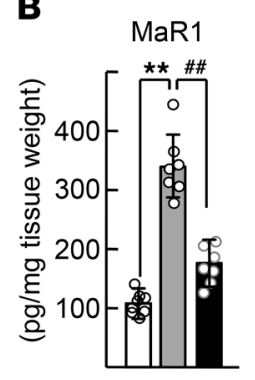

C

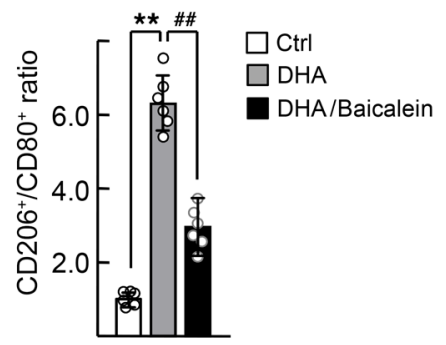

D
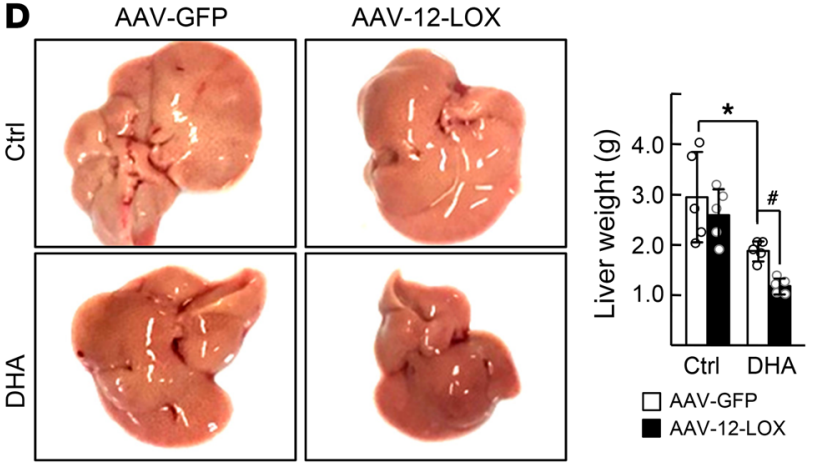

E

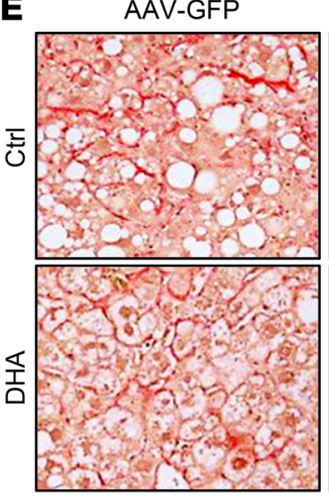

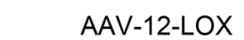

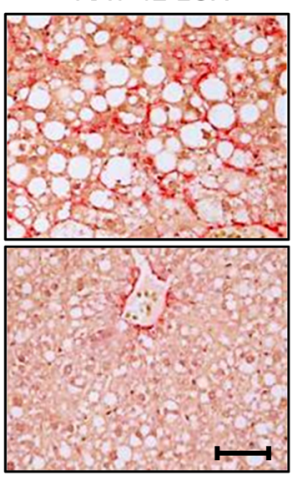

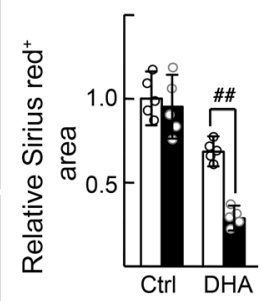

$\mathbf{F}$

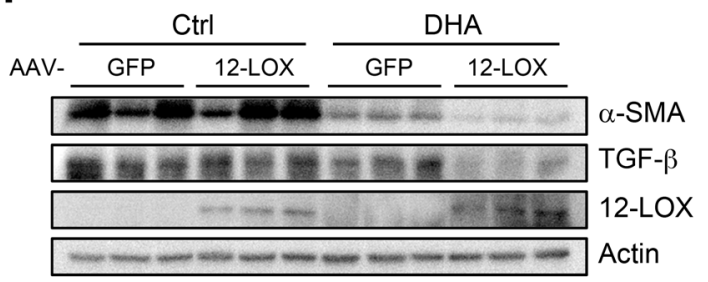

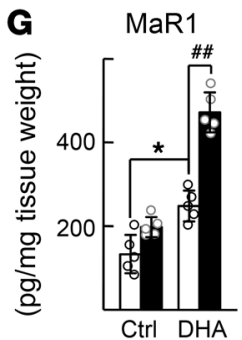

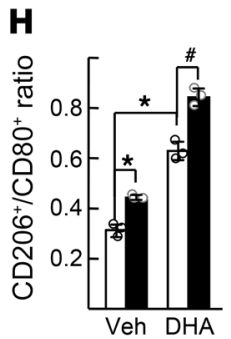

Figure 7. 12-LOX modulates the DHA-mediated improvement of NASH. (A-C) Seven-week-old WT male C57BL/6 mice were fed with HFD for 12 weeks. After 10 weeks of diet feeding, DHA was i.p. injected daily at dose of $5 \mathrm{mg} / \mathrm{kg}$ BW with vehicle or $5 \mathrm{mg} / \mathrm{kg}$ BW baicalein for 2 weeks. (A) Representative captured liver tissues and H\&E staining of liver sections. Scale bar: $200 \mu \mathrm{m}$. (B) Amount of MaR1 in the liver tissues were analyzed. (C) The liver macro-

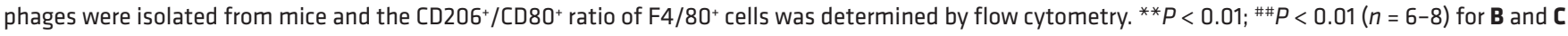
(D-C) Seven-week-old WT male C57BL/6 mice were fed with HFD for 16 weeks. After 13 weeks of diet feeding, an intravenous injection of either AAV-GFP or AAV-12-LOX at $5 \times 10^{9}$ virus genomes was conducted. DHA was i.p. injected daily at doses $1 \mathrm{mg} / \mathrm{kg}$ BW for 3 weeks after virus injection. (D) Representative captured liver tissues and the liver weights at the end of experiments. (E) Sirius red staining of liver sections. Fibrotic area in the liver sections was analyzed by Image J. Scale bar: $50 \mu \mathrm{m}$. (F) Levels of $\alpha$-SMA, TGF- $\beta$, and 12-LOX proteins in the liver tissues were analyzed by Western blotting. (C) The amount of MaR1 in liver tissues were measured. ${ }^{*} P<0.05$, ${ }^{\#} P<0.05$, and ${ }^{\# \#} P<0.01(n=5)$ for $\mathbf{D}-\mathbf{G}$. (H) Isolated liver macrophages were treated with 50 $\mu$ M DHA and then infected by AAV-GFP or AAV-12-LOX. The CD206 $/$ CD80 ${ }^{+}$ratio of F4/80 cells was determined. ${ }^{*} P<0.05$, ${ }^{*} P<0.05$ ( $n=3$ ). The data represent mean \pm SD. Data were analyzed by Mann-Whitney $U$ test for simple comparisons or Kruskal-Wallis test for multiple groups.

in the peripheral blood mononuclear cell-derived macrophages $(43,48)$. Similarly, MaR1 induced phagocytosis of the liver macrophages within 1 hour in our study (Supplemental Figure 2A). However, MaR1-induced phagocytosis did not require activation of ROR $\alpha$, which is largely different from the MaR1-induced M2 polarity switch (Supplemental Figure 2B). Thus, this short-term action of MaR1 (i.e., phagocytosis) may require other types of receptor(s). Indeed, it was reported that other SPMs such as RvD1 bind to GPR32, a surface G protein-coupled receptor, although such receptor(s) has not been found for MaR1 (49). Further study on the molecular details of MaR1-induced phagocytosis would help to characterize the diverse roles of MaR1 in innate immunity during the progression of NASH.

Recently, nuclear receptors have been targeted to develop new therapeutic strategies against NASH. A PPAR $/ \delta$ dual agonist, elafibranor (GFT-505), showed resolution of NASH without worsening fibrosis in a phase II controlled trial and is currently being assessed in 2000 patients in a phase III study (ClinicalTrials.gov, NCT02704403) (50). Obeticholic acid, a farnesoid X receptor agonist, was shown to reduce NAFLD activity score in a randomized phase IIb FLINT trial (51). Further evaluation of the efficacy and safety of this drug to seek FDA approval is ongoing 
A

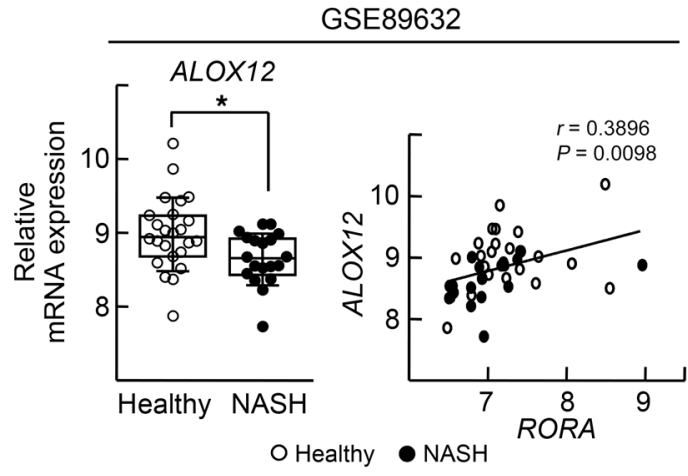

B

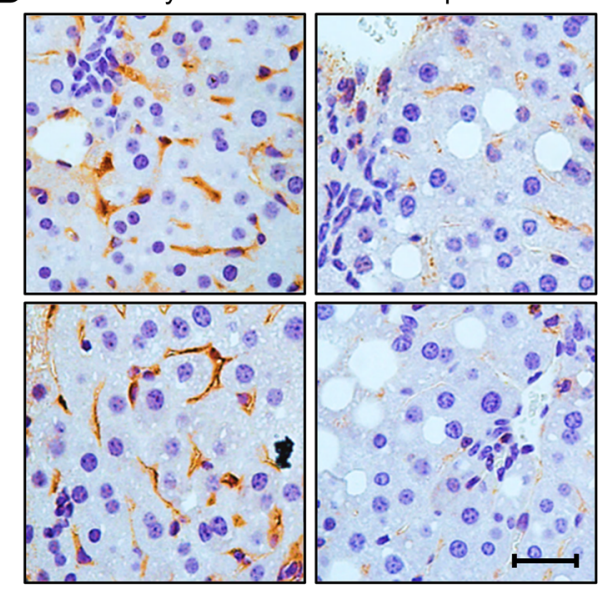

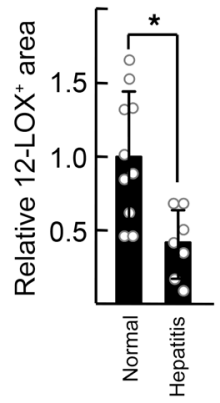
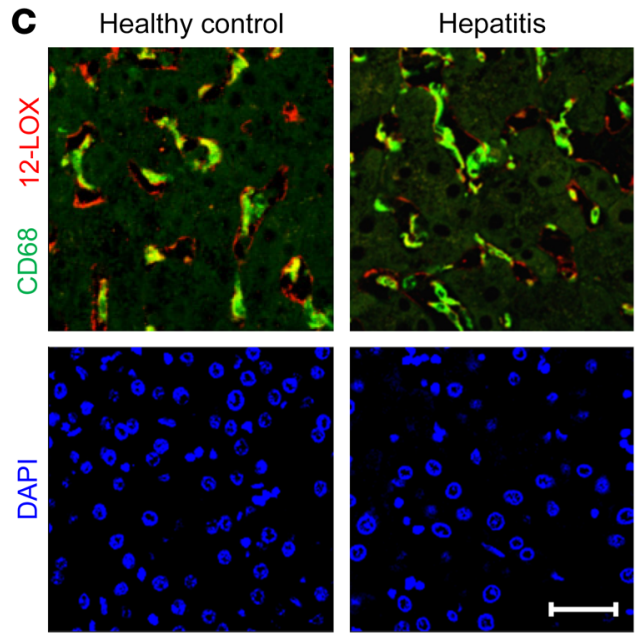

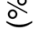

$\overbrace{}^{100}$
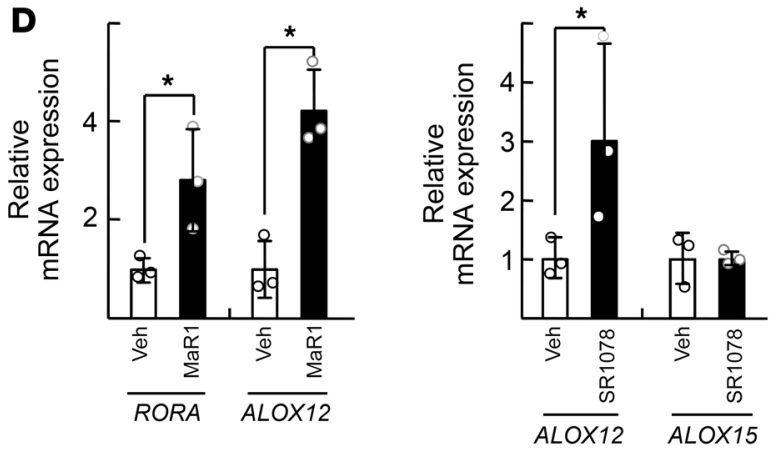

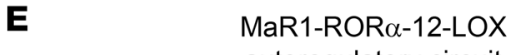

autoregulatory circuit
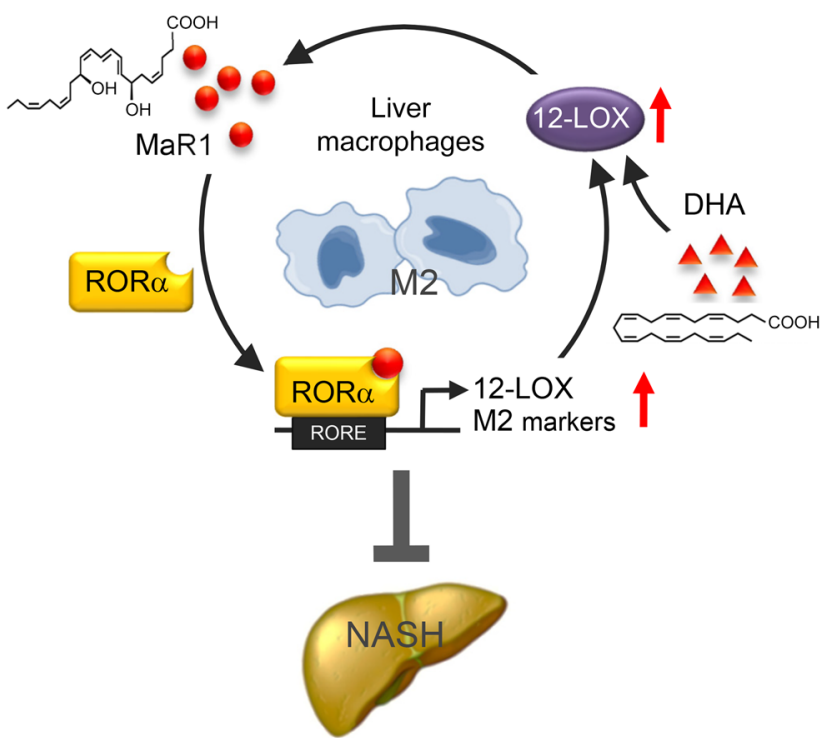

Figure 8. Expression of 12-LOX in liver macrophages is significantly low in the human patients with NASH. (A) Gene expression analysis was conducted using public data sets obtained from GEO site at the NCBI (http://www.ncbi.nlm.nih.gov/geo/). The data processed as quantile normalized intensity value. Significances were analyzed by Mann-Whitney $U$ test, and the positive correlation coefficient $(r)$ was calculated by Pearson correlation test for healthy controls $(n=24)$ and NASH patients $(n=19) ;{ }^{*} P<0.05$. (B) Immunohistochemistry staining of 12 -LOX in the liver specimens of the Biomax human tissue array is shown. The 12-LOX positive area was analyzed by Image J. ${ }^{*} P<0.05$ ( $n=10$ for healthy controls; $n=7$ for chronic hepatitis without B virus). Scale bar: $15 \mu \mathrm{m}$. For quantification, 8 fields of each specimen were analyzed. (C) Expression of 12-LOX and CD68 was visualized by red and green immunofluorescence in the liver specimens of the Biomax human tissue array. The nuclei were stained by DAPI that indicate nuclei of all the parenchymal and nonparenchymal liver cells. Colocalization of 12-LOX and CD68 were assessed by yellow colored dots that counted from at least 5 images per tissue using Image J software ( $n=17$ for healthy controls; $n=15$ for chronic hepatitis without B virus). ${ }^{*} P<0.01$. Scale bar: $40 \mu \mathrm{m}$. (D) THP-1 cells were treated with $200 \mathrm{nM}$ MaR1 or $5 \mu \mathrm{M}$ SR1078 for 24 hours. The mRNA levels of RORA, ALOX12, and ALOX15 were analyzed by qRT-PCR. ${ }^{*} P<0.05(n=3)$. The data represent mean \pm SD. Data were analyzed by Mann-Whitney $U$ test for simple comparisons. (E) Schematic model for the mechanism of MaR1/ROR $\alpha / 12-L O X$ autoregulatory circuit for M2 polarity switch in the liver macrophages. 
(ClinicalTrials.gov, NCT02548351). However, patients with mild NASH did not respond to either elafibranor or obeticholic acid, but showed side effects such as pruritus and decreased high-density lipoprotein cholesterol $(50,51)$. Thus, new strategies need to be established to overcome the limits of these nuclear receptor ligands. Recent findings support the pharmacological potential of FA mimetics with restricted toxicity and enhanced solubility and bioavailability as potential drug candidates for NASH (52). Here, the discovery of MaR1 as a strong ligand of ROR $\alpha$ that targets M2 polarization of liver macrophages could provide a good strategy to develop effective therapeutics for NASH. Effective and safe strategies that activate 12-LOX could enhance the autoregulatory activation loop of ROR $\alpha-12-\mathrm{LOX}$ through MaR1 synthesis.

In summary, we have demonstrated that the MaR1/ROR $\alpha / 12-$ LOX autoregulatory circuit can skew the polarity switch of liver macrophages, which could provide new insight into the clinical application of the nuclear receptors and lipid mediators for the treatment of NASH.

\section{Methods}

Cell culture and reagents. Liver macrophages were isolated from the liver of 7- to 10-week-old male C57BL/6 mice (Jackson Laboratory) by perfusion of liver using collagenase type IV (Sigma-Aldrich) as previously described (25). For isolation of liver macrophages, nonparenchymal sufficient supernatant was centrifuged in 50\%/25\% Percoll (GE Healthcare). The layer containing liver macrophages was plated with RPMI-1640 (Hyclone) with 10\% fetal bovine serum (FBS). The purity of liver macrophages exceeded $85 \%$ when estimated by either immunostaining or flow cytometry using anti-F4/80 (Santa Cruz Biotechnology, sc-52664), anti-FITC-F4/80 (eBioscience, catalog 11-4801), anti-PE-CD11b (eBioscience, catalog 12-0112), or anti-APC-Ly6C (eBioscience, 17-5932) antibodies (3). Raw 264.7 and THP-1 cell lines were obtained from American Type Culture Collection (ATCC) and cultured in Dulbecco's modified Eagle's medium (Hyclone) supplemented with 10\% FBS. Peritoneal macrophages were collected from the intraperitoneal cavity filled with PBS and cultured in RPMI-1640 with $10 \%$ FBS. The cells were grown in an incubator with $5 \% \mathrm{CO}_{2}$ and $95 \%$ air at $37^{\circ} \mathrm{C}$. Cells were incubated with DHA or MaR1 in free medium supplemented with 1\% FA-free BSA or 0.1\% FBS for 24 hours, respectively.

DHA, IL-4, Wy-14,643, troglitazone, and 9-cis-retinoic acid were purchased from Sigma-Aldrich. SR1078 was purchased from Tocris Bioscience. T0901317 was purchased from Alexis Biomedicas. MaR1, MaR2, protectin D1, RvD1, RvD2, and RvE1 were purchased from Cayman Chemical.

Plasmids, shRORa lentivirus, recombinant adeno-associated virus, and transient transfection. The RORE-tk-Luc and Gal4-tk-Luc reporter constructs were previously described (53). Eukaryotic expression vectors encoding C288L and $\mathrm{A} 330 \mathrm{~L} \mathrm{ROR} \alpha$ mutants were constructed by conventional gene recombination. The mouse Alox 12 promoters encoding regions -1355 approximately +294 and -1184 approximately +294 relative to the transcription start site were amplified by PCR and cloned into the Kpn I/Xho I site of the pGL3-basic vector. Lentiviral vectors encoding shGFP and shROR $\alpha$ were constructed using pLKO-TRC (Addgene). The lentiviruses were obtained by transient transfection of psPAX2 packaging plasmid and pMD2.G envelope plasmid in HEK293T cells. The AAVs encoding ROR $\alpha$ or 12-lipoxygenase (mouse 12-LOX; PlasmID DF/HCC DNA Resource Core, Harvard
Medical School) were obtained by transfection of pAAV-Myc-ROR $\alpha$, pAAV-12-LOX, pAAV-DJ, and pAAV helper in HEK293T cells. Seventy-two hours later, the AAVs in cell lysates were purified by using a ViraBind-AAV purification kit (Cell Biolabs). The AAV-12-LOX was generated and provided by SH Koo (Division of Life Sciences, Korea University, Seoul, Korea). Transduction procedures and knockdown efficiency of the lentivirus and AAV were previously described (3). Transient transfection to Raw 264.7 cells was carried out using Fugene HD transfection reagent (Promega).

Western blotting, coimmunoprecipitation, flow cytometry, ChIP, and ELISA. Western blotting and coimmunoprecipitation were performed as previously described using specific antibodies against ROR $\alpha$ (catalog sc-6062), p300 (catalog sc-585), NCoR1 (catalog sc-1609), TGF- $\beta$ (catalog sc-130348), actin (catalog sc-1616) (all from Santa Cruz Biotechnology); $\alpha$-SMA (catalog ab7817) and 12/15-LOX (catalog ab80221) (both from Abcam); NLRP3 (catalog 15101) and caspase 3 (catalog 9665)(both from Cell Signaling), or 12-LOX (catalog NBP229941; Novus Biologicals) (27). To analyze the M1/M2 status using flow cytometry, liver macrophages were stained with FITC-F4/80 (catalog 11-4801) and PE-CD80 (catalog 12-0801) (both from eBioscience), and Alexa647-CD206 (MCA2235A647; Serotec) after incubation with Fc $\gamma I I / I I I$ receptor antibodies (eBioscience, catalog 14-0161) to avoid nonspecific binding. To analyze intracellular ROR $\alpha$ protein levels in liver macrophages, cells were incubated with anti-ROR $\alpha$ antibodies (Thermo Fisher Scientific, catalog PA1-812) and then stained with combinations of PE-Cy5-F4/80 (eBioscience, catalog 15-4801) and anti-rabbit FITC-IgG (Serotec, catalog STAR121F) antibodies. Stained cells were analyzed with a FACS Calibur instrument and Cell Quest software (BD Bioscience). Some details of the method were previously described (3). The ChIP assay was carried out using goat anti-ROR $\alpha$ (catalog sc-6062), rabbit anti-p300 (Santa Cruz Biotechnology, catalog sc-585), or rabbit anti-histone acetyl K9 (Abcam, catalog ab4441) antibodies with specific primers as previously described (Supplemental Table 1) (3). The amounts of IL-10 and TNF- $\alpha$ protein were measured using commercial ELISA kits (AbFrontier) according to the manufacturer's protocol.

Quantitative real-time polymerase chain reaction ( $q R T-P C R)$. Total RNA was isolated using RNeasy Mini and Micro kits according to the manufacturer's protocol (Qiagen). qRT-PCR experiments were performed using an ABI StepOnePlus Real-Time PCR system with specific primers (Applied Biosystems) (Supplemental Table 1) (25). The relative mRNA level of target gene was estimated from the equation $2^{-\Delta C t}$ ( $\Delta C t=C t$ of target gene minus $C t$ of $\beta$-actin or $18 \mathrm{~S}$ rRNA). Fold inductions in the mRNA level of genes were presented with a control group level set at 1 .

Measurement of MaR1 and RvD1 level: LC/mass analysis. MaR1 and RvD1 were extracted with a mixture of $100 \mu \mathrm{g}$ cell lysates or $100 \mathrm{mg}$ liver tissue and $1.2 \mathrm{ml}$ methanol containing $500 \mathrm{pg} \mathrm{d}_{4}-\mathrm{LTB}_{4}$ internal standard. Samples were held at $-20^{\circ} \mathrm{C}$ for 45 minutes to allow for protein precipitation and then centrifuged ( $1200 \mathrm{~g}$ at $4^{\circ} \mathrm{C}$ for 10 minutes). SPMs were extracted using solid-phase extraction and eluted using methanol. Eluted isolates were then dried in a speed vacuum concentrator and suspended in methanol/water (80:20; vol/vol). LC-MS/ MS-based metabololipidomics was performed with a linear ion trap triple quadrupole mass spectrometer (Applied Biosystems, 3200 QTRAP) equipped with a HPLC system (Shiseido, HTS HPLC system) coupled to a LUNA C18 column $(2.0 \times 150 \mathrm{~mm}, 5.0 \mu \mathrm{m}$; Phenomenex $)$. The mobile phase consisted of methanol/ $\mathrm{H}_{2} \mathrm{O} /$ acetic acid in a ratio 
of 60:40:0.01 (vol/vol/vol) and ramped to 80:20:0.01 after 10 minutes and to 100:0:0.01 after 20 minutes. Instrument control and data acquisition were performed using Analyst software (Applied Biosyste$\mathrm{ms}$, version 1.5). Ion pairs from reported multiple reaction monitoring (MRM) methods were used for profiling and quantification of MaR1 and RvD1 (54). Quantification was carried out based on the peak areas obtained with MRM shifts and linear calibration curves for synthetic MaR1, RvD1, and $\mathrm{d}_{4}-\mathrm{LTB}_{4}$ for recoveries.

Ligand-binding analysis. FRET assays were performed using LanthaScreen TR-FRET ROR $\alpha$ coactivator assay (Invitrogen). The LBD of ROR $\alpha$ tagging GST and fluorescein-D22 coactivator peptide was incubated with a ligand for 20 minutes and fluorescence was detected with a multireader with background emission at $490 \mathrm{~nm}$ and binding signal at $520 \mathrm{~nm}$ after excitation at $340 \mathrm{~nm}$ (Molecular Devices). The ratio of emission signals at $520 \mathrm{~nm}$ and $490 \mathrm{~nm}$ was plotted.

SPR experiments were performed with a BIAcore 3000 system (GE Healthcare). The production and purification of the pET21a $\mathrm{a}^{+}$ GST-ROR $\alpha$-His was previously described (25). The GST-ROR $\alpha$-His proteins were immobilized onto the CM5 sensor chip (GE Healthcare). The solution of ligand dissolved in PBS-T with $0.01 \%$ ethanol was injected at a flow rate of $30 \mu \mathrm{l} / \mathrm{min}$. Affinity constants $\left(K_{D}\right)$ were calculated by using nonlinear fitting with the simple 1:1 Langmuir binding model by using BIAevaluation software (Biacore $A B$, version 3.1).

The docking study was performed in Sybyl-X 2.0 using the Surflex-Dock program. The crystal structure of the human ROR $\alpha$ in complex with cholesterol sulfate was downloaded from the Protein Data Bank (PDB ID: 1SOX) (28). Data for the ligand was put into a Sybyl database. Hydrogen atoms were added and minimized using the MMFF94s force field with MMFF94 charge by applying the conjugate gradient method with a $0.05 \mathrm{kcal} / \mathrm{mol} \AA$ A energy convergence criterion and a distance-dependent dielectric constant. Protomol, an idealized representation of a ligand that makes every potential interaction with the binding site, was generated based on the ligand mode. MaR1 was constructed with accurate configurations and energy was minimized and stored in the Sybyl database. The compounds in the Sybyl database were docked into the binding site by using Surflex-Dock based on the protomol developed earlier. The extracted cholesterol sulfate was employed as a reference molecule. The docking protomol could reproduce the position of cholesterol sulfate (stored in Sybyl database) in the binding site with 0.46 root-mean-square deviation of the heavy atoms $(\mathrm{C}, \mathrm{O}$, and $\mathrm{S})$ of the extracted cholesterol sulfate.

Animal studies. The transgenic fat-1 C57BL/6 mice were previously reported (55). Eight-week-old WT littermates and fat-1 transgenic mice were fed either HFD (D12942) or low-fat diet (LFD; D12450J) (Research Diets). The backcross, genotype, and validation of myeloid-specific ROR $\alpha$ knockout mice were previously described (3). The ROR $\alpha^{\mathrm{t} / \mathrm{l}}$ mutant embryo, which has loxP sites flanking exon 4 of the Rora gene, was provided from the Institut Clinique de la Souris (Illkirch-Graffenstaden, France). To generate the myeloid-specific ROR $\alpha$ knockout mice, $\mathrm{ROR} \alpha^{\mathrm{t} / \mathrm{l}}$ animals were cross-bred with LysM ${ }^{\text {Cre }}$ mice, which highly express Cre recombinase in myeloid cells by the lysozyme M promoter (Jackson Laboratories).

Seven-week-old male floxed or ROR $\alpha$-MKO mice were fed either HFD or LFD. MaR1 was administered daily at doses of $5 \mu \mathrm{g} / \mathrm{kg}$ body weight (BW) by intraperitoneal (i.p.) injection for 2 weeks. DHA (5\% BSA in PBS) was administered daily at doses of $5 \mathrm{mg} / \mathrm{kg}$ BW with or without $5 \mathrm{mg} / \mathrm{kg}$ BW baicalein by i.p. injection for 2 weeks. One intravenous injection of either AAV-GFP or AAV-12-LOX at $5 \times 10^{9}$ virus genomes was conducted 3 weeks prior to sacrifice. Seven-week-old WT male C57BL/6 mice were fed for 4 weeks with MCD diet or MCS diet as control (Dyets Inc). The groups of mice maintained a similar BW by feeding on the same type of diet. At the end of the experiments, liver tissues were excised and cross-sections of the left lobe of the liver were frozen for analyzing mRNA and SPMs levels, fixed in 10\% neutral buffered formalin (Sigma-Aldrich), or perfused by collagenase to collect immune cells. The activities of GPT and GOT in the serum were measured with a Fuji DRI-CHEM 3500s serum biochemistry analyzer (Fujifilm), and the amount of hepatic TG was measured with an EnzyChrom Triglyceride Assay Kit (BioAssay System). For histological examinations, 3- $\mu \mathrm{m}$ sections of paraffin-embedded tissue were stained with hematoxylin and eosin, Sirius red staining, and immunohistochemistry using anti-4-hydroxynonenal (JalCa, catalog MHN100P), anti-F4/80 (Santa Cruz Biotechnology, catalog sc-25830), and anti-CD206 (R\&D Systems, catalog AF2535) antibodies. Frozen liver tissue sections were stained with Oil Red O (Sigma-Aldrich).

Human tissue array. Liver disease spectrum tissue arrays that contain liver tissue sections from healthy individuals and patients with hepatitis were purchased from Biomax (catalog LV20812a). The slides were incubated with mouse anti-CD68 (Santa Cruz Biotechnology, catalog sc-20060) and rabbit anti-12-LOX (Novus Biologicals, catalog NBP2-29941) antibodies. The slides were then incubated with the secondary antibodies anti-rabbit IgG Alexa555 (catalog A31572) and antimouse IgG Alexa488 (Invitrogen, catalog A21200). DAPI was used to stain nuclei (as a control). The stained tissue samples were captured with a confocal microscope (Carl Zeiss).

Statistics. All values were expressed as mean \pm standard deviation. Statistical analysis was performed using nonparametric Mann-Whitney $U$ test for simple comparisons. Statistical analyses of multiple groups were conducted using 1-way Kruskal-Wallis test, except for Figure $2 \mathrm{E}$ which was analyzed using 2-way Bonferroni posttest. A $P$ value less than 0.05 was considered statistically different.

Study approval. All experiments with mice were performed in a blind and randomized fashion and approved by the Seoul National University Institutional Animal Care and Use Committee.

\section{Author contributions}

YHH and MOL designed the study and interpreted the results. $\mathrm{YHH}$, JYK, and HJK conducted most of the in vitro and in vivo experiments. KOS and YML measured the amount of MaR1 and RvD1. DBK, WJC, and BJL designed interaction modeling of ROR $\alpha$ and MaR1. JYC performed the study of the fat- 1 transgenic mice. YHH and MOL wrote the manuscript. MOL supervised the research.

\section{Acknowledgments}

We are grateful to J. Yu (Department of Chemistry \& Education, Seoul National University, Seoul, Korea) for consulting on BIAcore experiments. We thank SH Koo (Division of Life Sciences, Korea University, Seoul, Korea) for providing the AAV-12-LOX. This project was supported by grants from the National Research Foundation of Korea (2017R1A2B3011870 and 2018R1A5A2024425), and the Korea Mouse Phenotyping Project (NRF-2014M3A9D5A01073556).

Address correspondence to: Mi-Ock Lee, College of Pharmacy, Seoul National University, San 56-1 Sillim-dong, Kwanak-gu, Seoul 08826, South Korea. Phone: 82.2.880.9331; Email: molee@snu.ac.kr. 
1. Farrell GC, van Rooyen D, Gan L, Chitturi S. NASH is an inflammatory disorder: pathogenic, prognostic and therapeutic implications. Gut Liver. 2012;6(2):149-171.

2. Krenkel O, Tacke F. Liver macrophages in tissue homeostasis and disease. Nat Rev Immunol. 2017;17(5):306-321.

3. Han YH, et al. ROR $\alpha$ induces KLF4-mediated M2 polarization in the liver macrophages that protect against nonalcoholic steatohepatitis. Cell Rep. 2017;20(1):124-135.

4. Murray PJ. Macrophage polarization. Annu Rev Physiol. 2017;79:541-566.

5. Wan J, et al. M2 Kupffer cells promote M1 Kupffer cell apoptosis: a protective mechanism against alcoholic and nonalcoholic fatty liver disease. Hepatology. 2014;59(1):130-142.

6. Puri P, et al. A lipidomic analysis of nonalcoholic fatty liver disease. Hepatology. 2007;46(4):1081-1090.

7. Puri $\mathrm{P}$, et al. The plasma lipidomic signature of nonalcoholic steatohepatitis. Hepatology. 2009;50(6):1827-1838.

8. Parker HM, Johnson NA, Burdon CA, Cohn JS, O'Connor HT, George J. Omega-3 supplementation and non-alcoholic fatty liver disease: a systematic review and meta-analysis. J Hepatol. 2012;56(4):944-951.

9. Scorletti E, et al. Effects of purified eicosapentaenoic and docosahexaenoic acids in nonalcoholic fatty liver disease: results from the Welcome ${ }^{*}$ study. Hepatology. 2014;60(4):1211-1221.

10. Hodson L, et al. Docosahexaenoic acid enrichment in NAFLD is associated with improvements in hepatic metabolism and hepatic insulin sensitivity: a pilot study. Eur J Clin Nutr. 2017;71(10):1251.

11. Argo CK, et al. Effects of $\mathrm{n}-3$ fish oil on metabolic and histological parameters in NASH: a doubleblind, randomized, placebo-controlled trial. JHepatol. 2015;62(1):190-197.

12. Dasarathy S, et al. Double-blind randomized placebo-controlled clinical trial of omega 3 fatty acids for the treatment of diabetic patients with nonalcoholic steatohepatitis. JClin Gastroenterol. 2015;49(2):137-144

13. Huang SC, et al. Cell-intrinsic lysosomal lipolysis is essential for alternative activation of macrophages. Nat Immunol. 2014;15(9):846-855.

14. Li P, et al. NCoR repression of LXRs restricts macrophage biosynthesis of insulin-sensitizing omega 3 fatty acids. Cell. 2013;155(1):200-214.

15. Oishi Y, et al. SREBP1 contributes to resolution of pro-inflammatory TLR4 signaling by reprogramming fatty acid metabolism. Cell Metab. 2017;25(2):412-427.

16. Oh DY, et al. GPR120 is an omega-3 fatty acid receptor mediating potent anti-inflammatory and insulin-sensitizing effects. Cell. 2010;142(5):687-698

17. Talamonti E, Pauter AM, Asadi A, Fischer AW, Chiurchiù V, Jacobsson A. Impairment of systemic DHA synthesis affects macrophage plasticity and polarization: implications for DHA supplementation during inflammation. Cell Mol Life Sci. 2017;74(15):2815-2826.

18. Buckley CD, Gilroy DW, Serhan CN. Proresolving lipid mediators and mechanisms in the resolution of acute inflammation. Immunity. 2014;40(3):315-327.

19. Spite M, Clària J, Serhan CN. Resolvins, specialized proresolving lipid mediators, and their potential roles in metabolic diseases. Cell Metab. 2014;19(1):21-36.

20. Martínez-Fernández L, et al. Maresin 1 improves insulin sensitivity and attenuates adipose tissue inflammation in ob/ob and diet-induced obese mice. FASEB J. 2017;31(5):2135-2145.

21. Laiglesia LM, et al. Maresin 1 mitigates liver steatosis in ob/ob and diet-induced obese mice. Int $\mathrm{J}$ Obes (Lond). 2018;42(3):572-579.

22. Dalli J, Serhan CN. Specific lipid mediator signatures of human phagocytes: microparticles stimulate macrophage efferocytosis and pro-resolving mediators. Blood. 2012;120(15):e60-e72.

23. Jetten AM. Retinoid-related orphan receptors (RORs): critical roles in development, immunity, circadian rhythm, and cellular metabolism. Nucl Recept Signal. 2009;7:e003.

24. Ou Z, et al. Regulation of the human hydroxysteroid sulfotransferase (SULT2A1) by ROR $\alpha$ and ROR $\gamma$ and its potential relevance to human liver diseases. Mol Endocrinol. 2013;27(1):106-115.

25. Han YH, et al. ROR $\alpha$ decreases oxidative stress through the induction of SOD2 and GPx1 expression and thereby protects against nonalcoholic steatohepatitis in mice. Antioxid Redox Signal. 2014;21(15):2083-2094.

26. Kim EJ, et al. Retinoic acid receptor-related orphan receptor $\alpha$-induced activation of adenosine monophosphate-activated protein kinase results in attenuation of hepatic steatosis. Hepatology. 2012;55(5):1379-1388.

27. Kim HJ, et al. Liver-specific deletion of RORo aggravates diet-induced nonalcoholic steatohepatitis by inducing mitochondrial dysfunction. $\mathrm{Sci}$ Rep. 2017;7(1):16041.

28. Kallen J, Schlaeppi JM, Bitsch F, Delhon I, Fournier B. Crystal structure of the human RORalpha ligand binding domain in complex with cholesterol sulfate at 2.2 A.J Biol Chem. 2004;279(14):14033-14038.

29. Stehlin C, et al. X-ray structure of the orphan nuclear receptor RORbeta ligand-binding domain in the active conformation. $E M B O J$. 2001;20(21):5822-5831.

30. Kang JX, Wang J, Wu L, Kang ZB. Transgenic mice: fat- 1 mice convert n- 6 to n-3 fatty acids Nature. 2004;427(6974):504

31. Wang Y, et al. Identification of SR1078, a synthetic agonist for the orphan nuclear receptors ROR $\alpha$ and ROR $\gamma$. ACS Chem Biol. 2010;5(11):1029-1034

32. Zenri F, et al. Expression of retinoic acid-related orphan receptor alpha and its responsive genes in human endometrium regulated by cholesterol sulfate. J Steroid Biochem Mol Biol. 2012;128(1-2):21-28.

33. Bannenberg G, Serhan CN. Specialized proresolving lipid mediators in the inflammatory response: an update. Biochim Biophys Acta. 2010;1801(12):1260-1273.

34. Sekiya K, Okuda H. Selective inhibition of platelet lipoxygenase by baicalein. Biochem Biophys Res Commun. 1982;105(3):1090-1095.

35. Kenyon V, et al. Discovery of potent and selective inhibitors of human platelet-type 12- lipoxygen- ase. J Med Chem. 2011;54(15):5485-5497.

36. Rodriguez-Pacheco F, et al. Effects of obesity/ fatty acids on the expression of GPR120. Mol Nutr Food Res. 2014;58(9):1852-1860.

37. Ichimura A, et al. Dysfunction of lipid sensor GPR120 leads to obesity in both mouse and human. Nature. 2012;483(7389):350-354.

38. Quesada-López T, et al. The lipid sensor GPR120 promotes brown fat activation and FGF21 release from adipocytes. Nat Commun. 2016;7:13479.

39. Serhan CN, et al. Maresins: novel macrophage mediators with potent antiinflammatory and proresolving actions. J Exp Med. 2009;206(1):15-23.

40. Martínez-Clemente $\mathrm{M}$, et al. Disruption of the 12/15-lipoxygenase gene (Alox15) protects hyperlipidemic mice from nonalcoholic fatty liver disease. Hepatology. 2010;52(6):1980-1991.

41. Dobrian AD, Lieb DC, Cole BK, Taylor-Fishwick DA, Chakrabarti SK, Nadler JL. Functional and pathological roles of the 12- and 15-lipoxygenases. Prog Lipid Res. 2011;50(1):115-131.

42. Kutzner L, Goloshchapova K, Heydeck D, Stehling S, Kuhn H, Schebb NH. Mammalian ALOX15 orthologs exhibit pronounced dual positional specificity with docosahexaenoic acid. Biochim Biophys Acta Mol Cell Biol Lipids. 2017;1862(7):666-675.

43. Wang CW, et al. Maresin 1 biosynthesis and proresolving anti-infective functions with humanlocalized aggressive periodontitis leukocytes. Infect Immun. 2015;84(3):658-665.

44. Colas RA, Shinohara M, Dalli J, Chiang N, Serhan $\mathrm{CN}$. Identification and signature profiles for pro-resolving and inflammatory lipid mediators in human tissue. Am J Physiol, Cell Physiol. 2014;307(1):C39-C54.

45. Colas RA, et al. Identification and actions of the maresin 1 metabolome in infectious inflammation. J Immunol. 2016;197(11):4444-4452.

46. Titos E, et al. Resolvin D1 and its precursor docosahexaenoic acid promote resolution of adipose tissue inflammation by eliciting macrophage polarization toward an M2-like phenotype. JImmunol. 2011;187(10):5408-5418.

47. Chiang N, Dalli J, Colas RA, Serhan CN. Identification of resolvin $\mathrm{D} 2$ receptor mediating resolution of infections and organ protection. J Exp Med. 2015;212(8):1203-1217.

48. Dalli J, et al. The novel 13S,14S-epoxy-maresin is converted by human macrophages to maresin 1 (MaR1), inhibits leukotriene A4 hydrolase (LTA4H), and shifts macrophage phenotype. FASEB J. 2013;27(7):2573-2583.

49. Chiang N, Serhan CN. Structural elucidation and physiologic functions of specialized pro-resolving mediators and their receptors. Mol Aspects Med. 2017;58:114-129.

50. Ratziu V, et al. Elafibranor, an agonist of the peroxisome proliferator-activated receptor- $\alpha$ and $-\delta$ induces resolution of nonalcoholic steatohepatitis without fibrosis worsening. Gastroenterology. 2016;150(5):1147-1159.e5.

51. Neuschwander-Tetri BA, et al. Farnesoid X nuclear receptor ligand obeticholic acid for noncirrhotic, non-alcoholic steatohepatitis (FLINT): a multicentre, randomised, placebo-controlled trial. Lancet. 2015;385(9972):956-965.

52. Proschak E, Heitel P, Kalinowsky L, Merk D. 
Opportunities and challenges for fatty acid mimetics in drug discovery. JMed Chem. 2017;60(13):5235-5266.

53. Kim EJ, et al. Transcriptional activation of HIF- 1 by RORalpha and its role in hypoxia signaling. Arte- rioscler Thromb Vasc Biol. 2008;28(10):1796-1802. 54. Yang R, Chiang N, Oh SF, Serhan CN. Metabolomics-lipidomics of eicosanoids and docosanoids generated by phagocytes. Curr Protoc Immunol. 2011;Chapter 14:Unit 14.26.
55. Kim EH, Bae JS, Hahm KB, Cha JY. Endogenously synthesized $n-3$ polyunsaturated fatty acids in fat-1 mice ameliorate high-fat diet-induced non-alcoholic fatty liver disease. Biochem Pharmacol.2012;84(10):1359-1365. 